\title{
Testing Novel Portland Cement Formulations with Carbon Nanotubes and Intrinsic Properties Revelation: Nanoindentation Analysis with Machine Learning on Microstructure Identification
}

\author{
Georgios Konstantopoulos ${ }^{1}$, Elias P. Koumoulos ${ }^{1,2, * \mathbb{C}}$ and Costas A. Charitidis ${ }^{1}$ (1) \\ 1 RNANO Lab-Research Unit of Advanced, Composite, Nano Materials \& Nanotechnology, \\ School of Chemical Engineering, National Technical University of Athens, GR-15773 Zographos Athens, \\ Greece; gkonstanto@chemeng.ntua.gr (G.K.); charitidis@chemeng.ntua.gr (C.A.C.) \\ 2 Innovation in Research \& Engineering Solutions (IRES), Boulevard Edmond Machtens 79/22, \\ 1080 Brussels, Belgium \\ * Correspondence: epk@innovation-res.eu or elikoum@chemeng.ntua.gr
}

Received: 11 March 2020; Accepted: 27 March 2020; Published: 30 March 2020

\begin{abstract}
Nanoindentation was utilized as a non-destructive technique to identify Portland Cement hydration phases. Artificial Intelligence (AI) and semi-supervised Machine Learning (ML) were used for knowledge gain on the effect of carbon nanotubes to nanomechanics in novel cement formulations. Data labelling is performed with unsupervised ML with k-means clustering. Supervised ML classification is used in order to predict the hydration products composition and $97.6 \%$ accuracy was achieved. Analysis included multiple nanoindentation raw data variables, and required less time to execute than conventional single component probability density analysis (PDA). Also, PDA was less informative than ML regarding information exchange and re-usability of input in design predictions. In principle, ML is the appropriate science for predictive modeling, such as cement phase identification and facilitates the acquisition of precise results. This study introduces unbiased structure-property relations with ML to monitor cement durability based on cement phases nanomechanics compared to PDA, which offers a solution based on local optima of a multidimensional space solution. Evaluation of nanomaterials inclusion in composite reinforcement using semi-supervised ML was proved feasible. This methodology is expected to contribute to design informatics due to the high prediction metrics, which holds promise for the transfer learning potential of these models for studying other novel cement formulations.
\end{abstract}

Keywords: artificial Intelligence; machine learning; carbon nanotubes; cement microstructure; materials characterisation; nanoanalysis; nanomechanics

\section{Introduction}

Cement is considered as the most important hydraulic material in the modern construction field [1-4]. "Smart" sensing, self-healing, and self-sealing properties are in the spotlight and are engineered by nanomaterials addition [1,5], contributing also to materials design revolution for key industrial applications [1]. Since concrete is extensively studied [4], the design parameters are well-known in order to deliver suitable mechanical properties in the relevant application field. A plethora of new data are generated by characterization of novel cement formulations [6]; however, sufficient technology transfer to industry is scarce [1]. Except for the technology growth, synthesis of new materials and hybrid composite structures, the need to involve emerging evaluation methodologies is highlighted [1,7] to assist and accelerate developments [8,9]. Artificial Intelligence (AI) is a 
promising candidate to bridge the gap between Research and Development (R\&D) and industry by establishing unbiased relations of microstructure to properties [4,6-11]. This is majorly appreciated in case of Safe-by-Design requirements regarding mechanical performance [8,12], and real-time characterization [9]. Being representative, k-means, Random Forrest (RF), Support Vector Machines (SVM), k-Nearest Neighbors (KNN) are common Machine Learning (ML) algorithms used in multiclass classification problems [4] for automated classification of microstructures [8,13]; however, these algorithms often require lot of data to train the predictive models [14]. Also, density functional theory (DFT) has been established for predicting the structure and behavior of organic (such as proteins) and inorganic (i.e., most common are calcium carbonates, oxalates, metal sulfides, etc.) crystals, which has enabled the development of ontology databases; the calculated properties of known systems and the predicted properties of hypothetical systems are included $[10,14]$. Similar efforts have been put in practice with experimental materials characterization (CHADA, Nanoindentation-documentation structure for characterisation data) [11].

Grid nanoindentation is a highly localized and non-destructive technique with high spatial resolution [6,7]. It is a method that is suitable for fast and precise characterization of construction materials as concrete, metal alloys, coatings, and composites reinforced with micro- and nanomaterials $[3,6,7,9,13,15,16]$, being one of the few techniques that can directly assess the mechanical properties at micro- and nano- level by a single experiment $[6,17]$. Nanomechanical properties, and especially reduced Elastic modulus $\left(E_{r}\right)$, are involved in materials design and various set-ups $[18,19]$. These applications are very sensible in regards to the applied loads and are closely related to human and environmental safety. This input can be obtained in a representative manner, since statistical nanoindentation is able to characterize a surface via a multitude of indentation events [15]. Also, the contact surface is in the same scale of the characterized phases as in case of heterogeneous cement interface [20]. Grid size is usually sufficiently large, for instance when testing concrete, to encompass the various cement phases [6]. Quantification of the constituent volume fractions provides insight of the composite as a whole entity $[6,19]$. Thus, the generated data are suitable for statistical representation of nanomechanical properties of the tested material [6].

Focusing on the case of cement composites, a lot of effort is put in regards to nanoindentation [7]. Nanoindentation technique is gaining widespread attention due to the ability to both identify and quantify cement phases $[6,17,21]$. In detail, pores $E_{r}$ and hardness $(H)$ are derived by interfacial interaction with the low density (LD) boundary phases [22], and $E_{r}$ of hydrated phases is connected to degree of hydration $[4,23]$. Hardness is related to the yield strength of the hydrated cement phases, which are considered to behave as rigid cohesive plastic solids, granted that the size of interaction volume is smaller than indentation imprint. As part of this procedure, individual hydrated cement phases have been tested and processed via statistical analysis to determine $E_{r}$ and $H$ of these phases $[23,24]$, in order to provide the ability to connect mechanical properties to structure (density, crystallinity degree).

Portland Cement is used in every-day applications due to the low-cost, and workability. Calcium Aluminate Cements (CAC) facilitate protection from corrosion, temperature resistance, high strength, but are available at higher cost [25]. The main difference with ordinary Portland Cement lies in the active phase that is responsible for hardening due to the high aluminum content (up to $80 \mathrm{wt} . \%$ ) [26]; monocalcium aluminate (up to $46 \%$ ) is the active phase in CAC and yields into calcium aluminate hydrates (CAHs) formation instead of C-S-H [25]. In the present study, CEM I $52.5 \mathrm{~N}$ Portland Cement was used, which consists of $4.75 \mathrm{wt} . \% \mathrm{Al}_{2} \mathrm{O}_{3}, 19.47 \mathrm{wt} . \% \mathrm{SiO}_{2}$, and $63.16 \mathrm{wt} . \% \mathrm{CaO}$; the absence of aluminate hydrates is expected, considering the phase diagram of $\mathrm{CaO}-\mathrm{Al}_{2} \mathrm{O}_{3}-\mathrm{SiO}_{2}$ [26], thus C-S-H and $\mathrm{CaOH}$ (Portlandite) dominate [27]. Ettringite phase exists in the matrix phase mixed with Portlandite, at significantly lower ratios considering the sulfur role in ettringite formation and in the initial composition of CEM I $52.5 \mathrm{~N}[27,28]$; it is expected to comprise up to $\sim 5 \%$ combined with CAHs in the cement paste. The dominance of $\mathrm{C}-\mathrm{S}-\mathrm{H}$ is also reported in mixtures of Portland Cement and $<20 \mathrm{wt} . \%$ of $\mathrm{CAC}$, while alumina presence is restricted in the form of ettringite, calcium 
aluminomonosulfate, or $\mathrm{C}_{4} \mathrm{AH}_{\mathrm{x}}$ [26]. A short summary of material parameters determined by nanoindentation and correlation to cement phases is provided in Table 1.

Table 1. Material parameters determined by nanoindentation of hydrated cement phases.

\begin{tabular}{cccc}
\hline Cement Phase & $\boldsymbol{E}_{\boldsymbol{r}} \mathbf{( G P a )}$ & $\boldsymbol{H} \mathbf{( G P a )}$ & Reference \\
\hline Low stiffness phase & $0-13$ & $<0.4$ & {$[22,29-32]$} \\
Low density C-S-H & $7-34$ & $0.4-0.8$ & {$[2,3,15,18,19,22,23,29-41]$} \\
High density C-S-H & $25-39$ & $0.8-1.25$ & {$[3,15,18,19,22,23,29-32,36-38,40,42]$} \\
Portlandite (CH) & $<35$ & $1.31-1.66$ & {$[2,29-33,35-37,40,42]$} \\
Anisotropic Portlandite $(\mathrm{CH})$ & $\geq 99$ & $\geq 2.8$ & {$[21,23,31,41,43]$} \\
Clinker & $93-160$ & $3-10$ & {$[3,17,21,22,30,31,37,40,41]$} \\
\hline
\end{tabular}

The main target of this work is to identify each cement phase nucleation dependence on nano-reinforcement by carbon nanotubes (CNTs) by clustering material parameters determined by nanoindentation initially and by identifying the hydrated cement phases with k-nearest neighbors, support vector machines, and classification tree algorithms further on. Nanoindentation data analysis is used to train models for prediction of hydrated cement phases by using raw data as input. This step is essential to overcome exhaustive probability distribution fitting approach, in order to reach unbiased conclusions about phase composition. Till now, this approach involved the use of error minimization procedures, due to the non-uniqueness of the solution [44] and depends on the selection of initial values [6], i.e., when fitting five phases with five Gaussians, a 15-dimensional (3 Gaussian parameters $\times 5$ phases) is created and the solution represents one of the local optima [43]. Also, skewness of fitting is usually omitted by nullifying the third and fourth statistical moments in order to simplify analysis [38]. This is a task of predictive modeling, which was performed using statistics in the past decades due to insufficient computational power. Predictive modeling is rapidly growing, due to the availability of computational resources, and better results are obtained with implementation of ML [45]. Consequently, structure-property relations will enhance objectivity and knowledge-gain for decision making by the incorporation of ML.

The matrix area of Portland Cement was selected for characterization, considering the fact that $\mathrm{C}-\mathrm{S}-\mathrm{H}$ is the major contributor in the final properties and durability of hardened cement [27]; measure nanomechanical properties in the interface transition zone to monitor phases nucleation. To our knowledge, this is the first time that cement mechanical properties are processed with ML to monitor the microstructure evolution and establish unbiased structure-property relations. Except for image classification (i.e., from Scanning Electron Microscopy, X-ray Tomography) and elemental analysis (i.e., from Energy Dispersive X-Ray Spectroscopy-EDX) no other data have been processed with ML algorithms to date, in order determine the cement and concrete hydrated phases quantitatively $[4,17,31,46]$. Specifically, the spatial deconvolution of Calcium(-Silicate)-Hydrates (C-S-H) of lower and higher density is not yet envisioned with AI, while there is no feedback for the interface effect induced by the neighboring clinker regions; image analysis of these regions is restricted by the color definition, which is the same for C-S-H and interfacial effect of clinker may be considered as Portlandite (pixels attributed to clusters) [31]. This fact hinders any straightforward connection of hydration progress and hydrated cement phases; this challenge is met by involving nanomechanics and supported further by the prediction metrics, which are exceeding relevant reported values for identification of individual phases of cement matrix using nanoindentation compared to image data analysis. The methodology implementation for phase identification in cement is expected to enhance analysis of testing ordinary Portland Cement by the transfer learning potential of the developed models. It is also expected to contribute to testing other formulations or concrete; similar principles are involved, along with data preprocessing, the use of classification algorithms by making several adaptations. 


\section{Materials and Methods}

\subsection{Nanocomposite Manufacturing}

Specimens received, were formulated using Portland Cement (CEM I 52.5N, Lafarge Beton S.A., Paiania, ATH, Greece), CEN Standard sand (Normensand GmbH, Beckum, Germany), and distilled water (EN 196 standard). Carbon nanotubes were synthesized through Chemical Vapor Deposition method (CVD); synthesis and chemical modification process is described in detail in previous work $[5,47]$. The wet-mixing method was used for nanocomposites molding with $0.02 \%, 0.05 \%, 0.1 \%$, $0.2 \%, 0.5 \%$, and $1 \%$ CNTs by weight of cement (bwoc), and water to cement fraction was w/c equal to 0.5. The sand/cement ratio in mortar specimens was 3:1, while curing was performed in saturated atmosphere of $95 \%$ humidity, which was controlled by using saturated $\mathrm{KNO}_{3}$ aqueous solution. All the aspects of manufacturing, and hardening of nanocomposites are described in the relevant work of Karaxi et al. [5].

\subsection{Grid Nanoindentation}

The nanoindentation tests were performed using a Hysitron (Minneapolis, MN, USA) TriboLab ${ }^{\circledR}$ Nanomechanical Test Instrument equipped with a Berkovich diamond indenter (average radius $100 \mathrm{~nm}$ ), which allows the application of loads from 1 to $30,000 \mu \mathrm{N}$ and records the displacement as a function of applied loads with a high load resolution $(1 \mathrm{nN})$ and a high displacement resolution (0.04 nm). Details about the instrument and the experimental setup have been presented elsewhere [16]. Maximum indentation depth was set at $200 \mathrm{~nm}$ in accordance to restrictions to satisfy the separability scale condition $\left(d / 10<<h_{\max }<<D / 10, d\right.$ and $D$ stand for the characteristic sizes of the largest heterogeneity) of cement phases $[17,29,32,38]$. Prior to indentation, the area function of the indenter tip was calibrated in a fused silica, a standard material for this purpose. All nanoindentation tests were conducted in a clean area environment with $45 \%$ humidity and $23{ }^{\circ} \mathrm{C}$ ambient temperature with displacement feedback control closed loop. All the aforementioned details comply with all nano range testing specifications as reported in ISO 14577-1:2015 for instrumented indentation. The volume of interest included the matrix region of cement (C-S-H, CH, and interface) similarly to Figure 1.

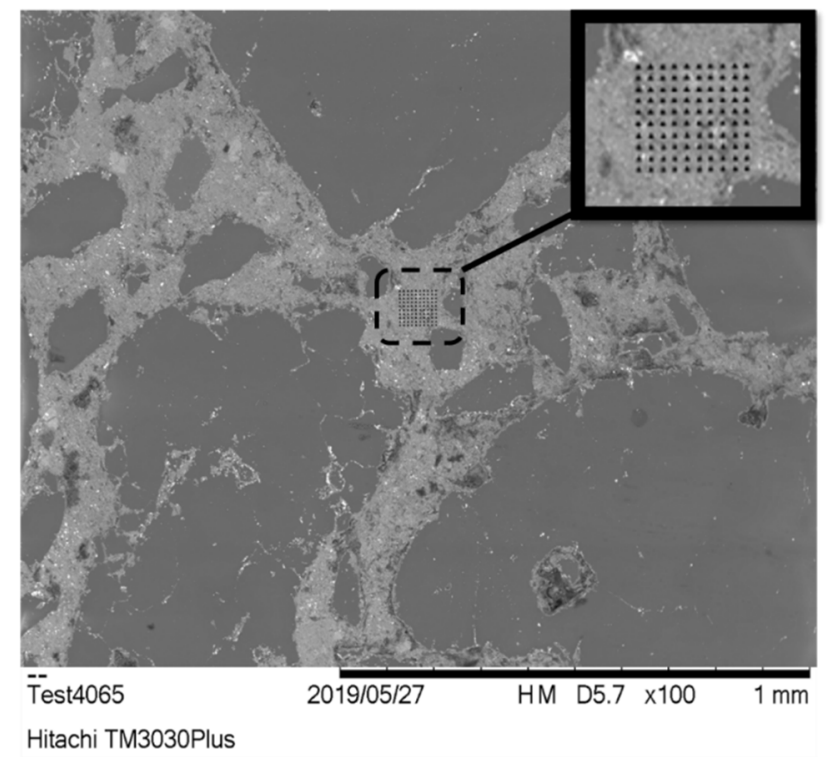

Figure 1. Guide for the eye: nanoindentation grid (in inset) in the interface area of Portland Cement.

Specimen preparation follows flat surface requirements for testing; a smooth surface was obtained by a wet polishing procedure using ethanol [48]. The used granulometry was a sequence of 400, 1000, 1200, 2000, and $4000 \mathrm{SiC}$ grinding papers for $10 \mathrm{~min}$ each, by Struers LaboPol-2 grinding, 
lapping, and polishing apparatus. The specimens were dried before testing at $125{ }^{\circ} \mathrm{C}$ to prevent further progress of hydration reactions, in order to remove humidity and water captured within crystal lattice [49]. Nanoindentation testing was performed with adequate spacing of $5 \mu \mathrm{m}$ to avoid any indentation-to-indentation interaction [7] and characterize individual cement phases [35,41]. The indenter was selected to probe at $200 \mathrm{~nm}$ of displacement. Material parameters determined by nanoindentation were measured via fitting with Oliver-Pharr model using the elastic response within the region of maximum load of unload curves [50].

\subsection{R Language}

R Language was implemented in R studio. R (version 3.6.0, R Core Team (2019). R: A language and environment for statistical computing. R Foundation for Statistical Computing, Vienna, Austria. URL https://www.R-project.org/) is an open-source software to use and provides a coherent, flexible system for data analysis. k-means algorithm was used for unsupervised data clustering. This was implemented as a labeling step to prepare data for classification. Phase correlation to material parameters determined by nanoindentation is usually a part of statistical PDA analysis, which is also performed via $\mathrm{R}$ for comparison. Then, the labeled data were used as a library to train classification models of RF, SVM, and KNN in order to evaluate the best predictive performance. All statistical calculations were performed using 64-bit Windows 10 Home (Intel ${ }^{\circledR}$ Core $^{\mathrm{TM}}$ i5-8250U CPU @ $1.60 \mathrm{GHz}$, 1801Mhz 4 Cores, 8 Logical Processors and 8.00 GB RAM). Computational time did not exceed a few seconds in each case. The session info and the R-packages are summarized in Table A1.

\subsection{Statistical Metrics}

In order to evaluate the prediction efficiency of the trained models, statistical metrics are involved. Accuracy, Precision, Recall, F1 were exported in each case [4,51-53], after tuning each model to the optimum in regard to accuracy by performing grid parameterization. Accuracy accounts for overall model accuracy. These metrics are maximized when the model does not generate false positive or false negative predictions as can be observed below:

$$
\begin{gathered}
\text { Accuracy }=\frac{T P+T N}{T P+T N+F P+F N} \\
\text { Recall }=\frac{T P}{T P+F N} \\
\text { Precision }=\frac{T P}{T P+F P} \\
\text { F1 Score }=2 \times \frac{\text { Precision } \times \text { Recall }}{\text { Precision }+ \text { Recall }}
\end{gathered}
$$

True positives (TP) denote correct classifications of Portland Cement phases (positive sample), true negatives $(T N)$ denote correct classifications of negative samples, false positives $(F P)$ denote the incorrect classifications of negative samples into positive samples, and false negatives (FN) denote the positive samples incorrectly classified into negative samples. Recall is the percentage of positive samples which are correctly classified. Precision is the percentage of positive samples out of the sum of positive observations. F1 score is a metric to evaluate the model ability to classify (best value: 1 ). Micro-metrics are expected to obtain the same value because there is only one class associated with each instance [52]. MacroAvgPrecision, MacroAvgRecall, MacroAvgF1 are mean values for overall model metrics. MicroAvgPrecision, MicroAvgRecall, MicroAvgF1 are metrics derived by the sum of the individual true positives, false positives, and false negatives of the system for different sets. 


\section{Results: Prediction of Portland Cement Composition}

\subsection{Data Preprocessing}

Nanoindentation raw data obtained from eight different Portland Cement specimens (reference and reinforced with CNTs) were merged using $\mathrm{R}$ language to a total of 790 indentations. Data were normalized in order to export the correlation matrix between the total nine variables. The recorded variables are:

- $h_{c}:$ is the contact depth,

- $P_{\text {max }}$ : is the peak load during a single nanoindentation event,

- $S:$ is a continuous variable and represents the stiffness of a material,

- $A$ : is the contact area

- $E_{r}$ : is the reduced elastic modulus after fitting the Oliver-Pharr model

- $H$ : is the hardness after fitting the Oliver-Pharr model

- $A, h_{f}, m$ : are the power law coefficients

$h_{c}(\mathrm{~nm})$ ranged between 11 and $20, P_{\max }(\mu \mathrm{N})$ ranged between 0 and $11, S(\mu \mathrm{N} / \mathrm{nm})$ between 0 and $7, A\left(\mathrm{~nm}^{2}\right)$ ranged between 3 and 11, $E_{r}(\mathrm{GPa})$ between 0 and $6.5, H(\mathrm{GPa})$ between 0 and $10, h_{f}(\mathrm{~nm})$ between 0 and $6, A$ between 0 and 11, and $\mathrm{m}$ between 1 and 7.5. The use of variables without strong correlation is necessary to avoid overfitting during training the classification models, and thus optimize computational time requirement. Thus, variables with positive or negative Pearson correlation exceeding the value of $R^{2}= \pm 0.90$ were excluded from analysis due to very strong correlation (Figure 2) [54]. A very strong correlation (>0.90) of variables means that the model based on training data provides a very accurate fit, which also complicates the patterns behind the fitting (termed as overfitting) and this usually hinders prediction of unknown testing datasets.

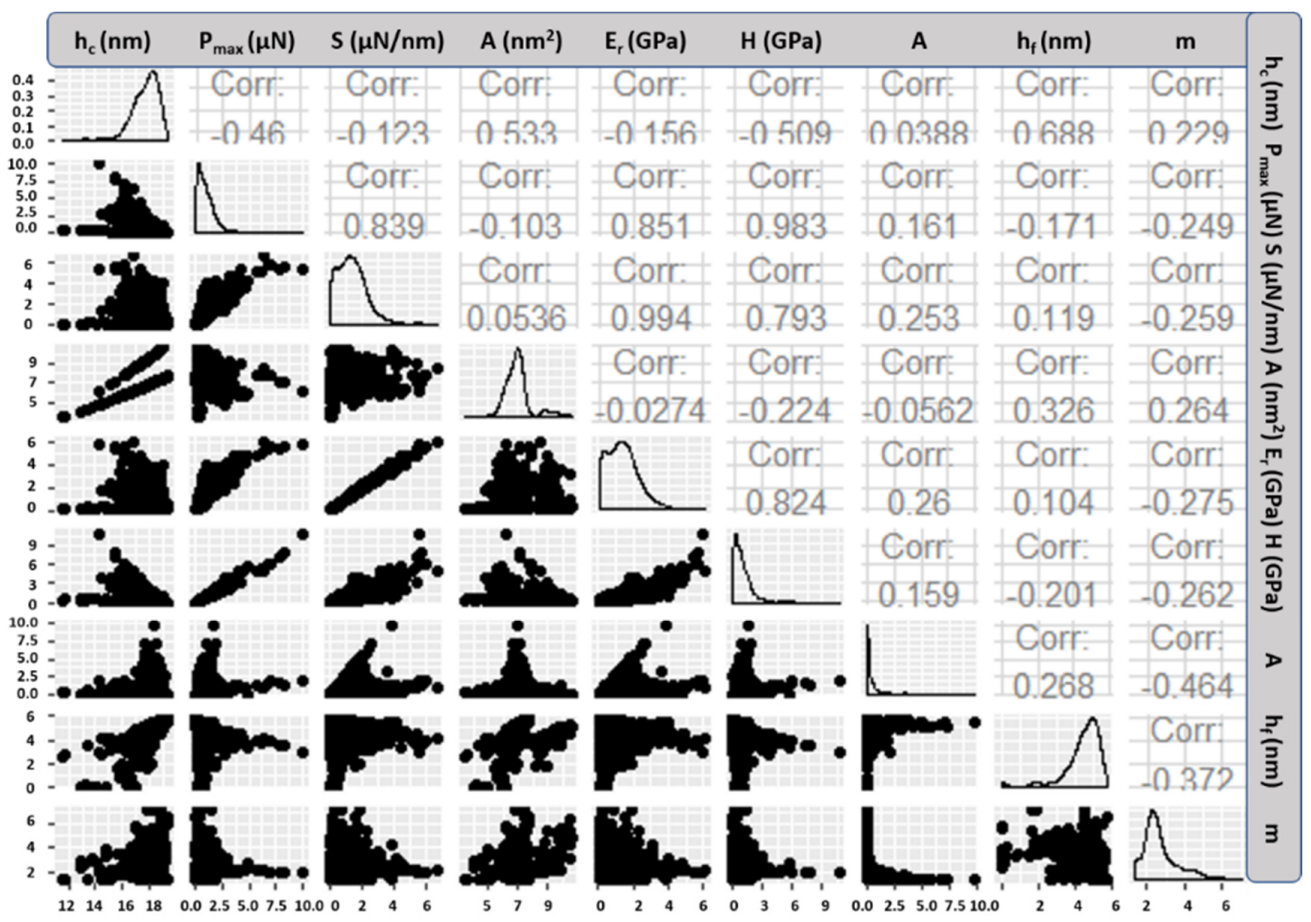

(a)

Figure 2. Cont. 


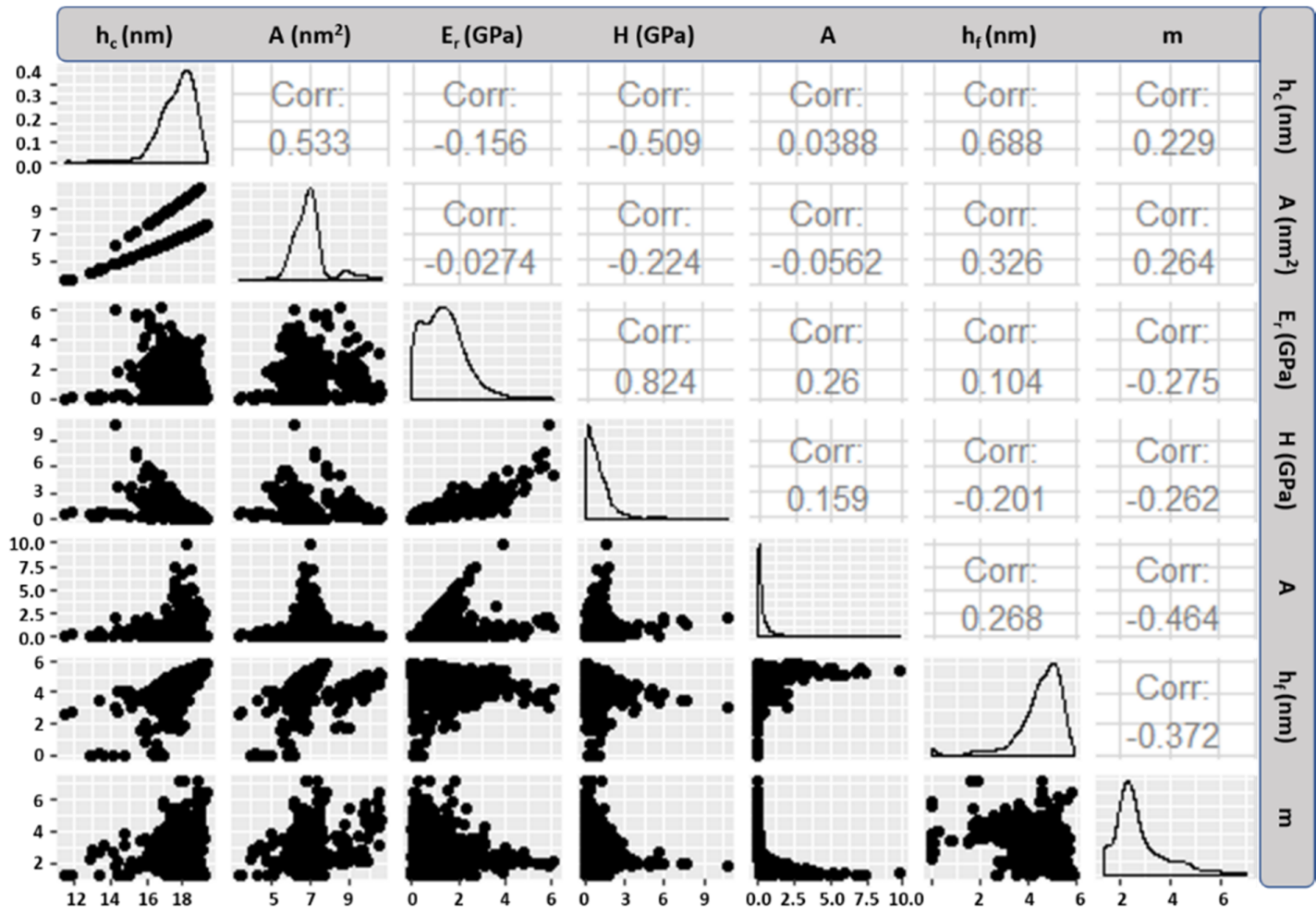

(b)

Figure 2. Correlation matrix of nanoindentation data prior (a) and after (b) data preprocessing. The scatter plots correspond to each combination of variables by two.

All the other variables were retained as special features of each cement phase will contribute to the identification problem during training of classification models. Then data were split to $80 \%$ train and $20 \%$ test datasets for classification models training and testing similar to [55].

\subsection{Data Labeling}

K-means algorithm was used to perform unsupervised ML and correlate material parameters (determined by nanoindentation) to Portland Cement phases. The number of clusters should be selected with pure probabilistic basis [46]. Thus, a multitude of criteria were incorporated. By applying the elbow method, the Bayesian inference criterion, and the Humbert criterion, the optimal number of clusters was estimated (Figure 3) [46,56]. Knowledge about the physical problem (Table 1) is still necessary since the aforementioned criteria demonstrate a different optimum number of clusters. The background of cement microstructure that is expected to be present within the characterized region (Figure 1) is solid. Taking into account that clustering within this study is performed for assigning labels on nanoindentation data in the framework of semi-supervised Machine Learning [57], the expected number of phases is 5 in the interface region of Portland Cement.

As depicted in Figure 3, it is proposed that optimum number of clusters is 4 (Figure 3a), but this is not confirmed by Bayesian inference criterion (BIC, Figure 3b) [46]. The maximum BIC value provides evidence for the number of existent constitutive phases in the dataset $[2,6,58]$, which is number 5 . The Humbert criterion correlates the highest frequency to the optimum number of clusters. Since 4 clusters are not sufficient to describe the number of cement phases, the next acceptable candidate is 5 clusters as indicated by the physical problem (Table 1). These clusters are attributed to LD C-S-H, including their presence in (gel) pores network (with lower stiffness), high density (HD) C-S-H, CH and its anisotropic configurations, and clinker interface (Figure 3e). At high w/c ratios (typically 
50:50 similar to the present study), UHD C-S-H is absent and $\mathrm{CH}$ phase is formed (only), since their nucleation mechanism is antagonistic [24,42].
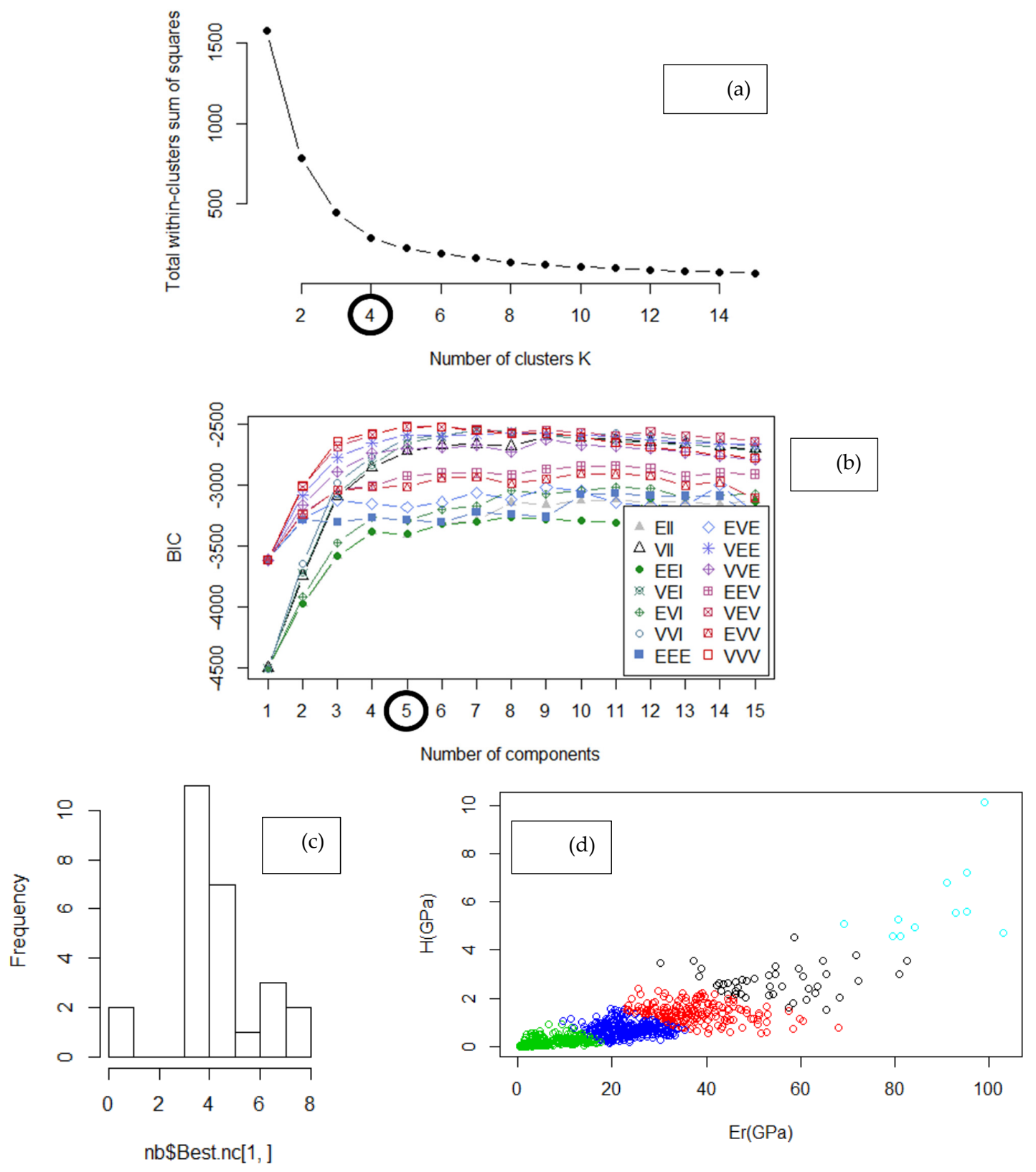

Figure 3. Cont. 


\begin{tabular}{|c|c|c|c|c|c|c|c|c|c|}
\hline$\approx$ & File & $\mathbf{h c}(\mathbf{n m})=$ & $\mathrm{A}\left(\mathrm{nm}^{\wedge} 2\right)$ & $\operatorname{Er}(\mathrm{GPa})$ & $\mathrm{H}(\mathrm{GPa})$ & A & $h f(n m)=$ & & cluster \\
\hline 453 & cementCNTs_05wt & 177.4928 & 678174.4 & 82.712695 & 3.555172 & 6.583318 & 153.154159 & 1.526074 & $\mathrm{CH}$ anisotropic \\
\hline 673 & mortarCNTs_01wt & 177.5236 & 678364.5 & 36.501314 & 1.551090 & 5.097977 & 157.038015 & 1.410624 & $\mathrm{CH}$ \\
\hline 457 & cementCNTs_05wt & 177.6033 & 678855.9 & 36.705521 & 1.570879 & 1.036223 & 146.773749 & 1.736806 & $\mathrm{CH}$ \\
\hline 475 & cementCNTs_05wt & 177.6037 & 678858.5 & 44.873511 & 1.861552 & 11.995194 & 161.711034 & 1.274792 & $\mathrm{CH}$ \\
\hline 645 & mortarCNTs_01wt & 177.6325 & 679036.3 & 10.241299 & 0.621078 & 0.000135 & 75.856418 & 3.048651 & LD C-S-H \\
\hline 126 & cement_ref & 177.6457 & 679118.1 & 15.252067 & 0.641518 & 1.317680 & 154.257717 & 1.511564 & HD C-S-H \\
\hline 246 & cementCNTs_002wt & 177.7150 & 679545.5 & 29.803157 & 1.268619 & 10.697676 & 163.413379 & 1.210014 & $\mathrm{CH}$ \\
\hline 746 & mortarCNTs_02wt & 177.8440 & 680342.7 & 1.534184 & 0.067750 & 0.000002 & 87.051305 & 3.563347 & LD C-S-H \\
\hline 192 & cement_ref & 177.9033 & 680709.1 & 19.192237 & 0.779071 & 1.686598 & 155.294663 & 1.511917 & HD C-S-H \\
\hline 104 & cement_ref & 177.9755 & 681155.7 & 15.932030 & 0.663429 & 2.248912 & 157.866725 & 1.410397 & HD C-S-H \\
\hline 516 & cementCNTs_1wt & 178.0243 & 681457.0 & 34.503466 & 1.407768 & 4.194519 & 157.325293 & 1.443627 & $\mathrm{CH}$ \\
\hline 310 & cementCNTs_005wt & 178.1476 & 682220.2 & 26.659195 & 1.071116 & 2.225581 & 155.395204 & 1.523821 & HD C-S-H \\
\hline 661 & mortarCNTs_01wt & 178.2433 & 682812.3 & 5.338380 & 0.303714 & 0.000019 & 72.329787 & 3.292788 & LD C-S-H \\
\hline 151 & cement_ref & 178.2531 & 682873.5 & 18.550138 & 0.750545 & 4.199786 & 161.590518 & 1.312482 & HD C-S-H \\
\hline 780 & mortarCNTs_02wt & 178.3095 & 683222.8 & 19.005229 & 0.948619 & 0.000530 & 96.549407 & 2.986695 & HD C-S-H \\
\hline 365 & cementCNTs_005wt & 178.3732 & 683617.3 & 1.043290 & 0.042671 & 0.026429 & 147.945109 & 1.765561 & LD C-S-H \\
\hline 613 & mortarCNTs_01wt & 178.4112 & 683852.3 & 7.581176 & 0.317632 & 0.000163 & 106.394557 & 3.096006 & LD C-S-H \\
\hline 233 & cementCNTs_002wt & 178.4133 & 683865.7 & 45.676523 & 1.863564 & 2.644380 & 153.068004 & 1.597864 & $\mathrm{CH}$ \\
\hline 132 & cement_ref & 178.4294 & 683965.5 & 14.157966 & 0.569252 & 0.794005 & 153.203856 & 1.606220 & HD C-S-H \\
\hline
\end{tabular}

Figure 3. Determination of optimum number of clusters (noted in circle for $\mathbf{a}, \mathbf{b}$ ) with (a) the elbow method, (b) Bayesian inference criterion, (c) Humbert criterion, and (d) $H$ vs. $E_{r}$ plot of clustered data by k-means algorithm, (e) labelled dataset. Symbols in (b): "EII": spherical, equal volume, "VII": spherical, unequal volume, "EEI": diagonal, equal volume and shape, "VEI": diagonal, varying volume, equal shape, "EVI": diagonal, equal volume, varying shape, "VVI": diagonal, varying volume and shape, "EEE": ellipsoidal, equal volume, shape, and orientation, " $E V E$ ": ellipsoidal, equal volume and orientation, "VEE": ellipsoidal, equal shape and orientation, " $V V E$ ": ellipsoidal, equal orientation, " $E E V$ ": ellipsoidal, equal volume and equal shape, " $V E V$ ": ellipsoidal, equal shape, " $E V V$ ": ellipsoidal, equal volume, " $V V V$ ": ellipsoidal, varying volume, shape, and orientation.

\subsection{Probability Distribution Analysis-PDA}

The most common approach to analyze nanoindentation data, especially in case of multiphase materials, is the application of Probability Density Function (PDF) that is intuitive regarding the identified histogram of material parameters (determined by nanoindentation) and the density plot. As a result, individual phases can be distinguished in the graph. In order to allocate the data in cement phases, a mixture model fit was selected based on Fraser-Suzuki function. The gain compared to conventional Gaussian-type normal distribution used in PDA is that the Fraser-Suzuki function allows for asymmetry [59], and that it is possible to provide a more appropriate fitting considering that neither measurement or the material are perfect $[38,60]$. The equation that describes the phase contribution to the overall PDF is presented below [59]:

$$
P D F=p_{i} \exp \left(-\frac{\ln 2}{s_{i}^{2}} \ln ^{2}\left(1+2 s_{i} \frac{E_{r}-E_{m, i}}{d_{i}}\right)\right)
$$

where $E_{r}$ is the reduced elastic modulus in $\mathrm{GPa}, \mathrm{p}_{\mathrm{i}}$ is the peak value of probability for each individual phase, $s_{i}$ is the skew of the fitting curve, $E_{m, i}$ is the mean reduced elastic modulus value of each individual phase, and $d_{i}$ is the width, an input value correlated to deviation from the mean value for each phase.

The phases have been identified by the use of nanoindentation to plot histograms of material parameters as for instance $E_{r}$ or $H[3,7,15,17,29,31,37-39,43]$ and by using the number of peaks in the density plot to manually select the number of phases to deconvolute (Figure 4a) $[3,29,32,43]$. 
All parameters of bin size, volume fraction, and initial mean values are all determined by the analyst prior to using PDF for fitting the data (Figure 4a) [3,38,39]. Nanoindentation data are preprocessed by the analyst to clean the error values, such as indentations that overcame the predefined maximum depth of $200 \mathrm{~nm}$. The model estimated a total of $20(4 \times 5)$ parameters in order to describe the 5 components of Portland Cement phases. Then minimization of PDF is evaluated whether the results of mean values, standard deviation, and volume fraction are descriptive for the analysis. If not, then initial values are readjusted and analysis is performed again [2,24,31]. In order to minimize the deviation between theoretical and empirical PDF, two approaches are adopted: least squares estimation (LSE) and maximum likelihood estimation (MLE-bin size is not selected manually) [2,24,61]. Starting values were selected based on nanoindentation studies on cement phase deconvolution [41]. For this purpose, an application for quick PDF was developed within this work [62]. The optimum parameters were selected based on the theoretical PDF, analyst decisions, and are presented in Figure 4. The probability distribution was measured by the integral of Equation (5). The respective fitting of cumulative distribution function is presented in Figure 4 for completeness. It was considered purposeful to compare phase identification using PDF to k-means clustering result. k-means clustering is unbiased by the analyst choices since the physical problem is well studied, and accepts input from multiple variables (in this case $E_{r}$ and $H$ ).

Table 2. Descriptive statistics of Portland Cement phases by implementing Probability Density Function (PDF) and k-means analysis.

\begin{tabular}{|c|c|c|c|c|c|c|c|c|c|c|c|}
\hline & $\begin{array}{c}\text { PDF } \\
\text { Probability } \\
\text { Peak }\end{array}$ & $\begin{array}{l}\text { PDF } \\
\text { skew }\end{array}$ & $\begin{array}{c}\text { PDF } \\
E_{r} \\
\text { (GPa) }\end{array}$ & $\begin{array}{c}\text { Deviation } \\
( \pm \text { GPa })\end{array}$ & $\begin{array}{c}\text { PDF } \\
\text { Volume } \\
\text { Fraction }\end{array}$ & $\begin{array}{l}\text { k-Means } \\
H \text { (GPa) }\end{array}$ & $\begin{array}{c}\text { k-Means } \\
\text { Deviation } \\
\text { ( } \pm \text { GPa) }\end{array}$ & $\begin{array}{l}\text { k-Means } \\
E_{r}(\mathrm{GPa})\end{array}$ & $\begin{array}{c}\text { k-Means } \\
\text { Deviation } \\
( \pm \text { GPa })\end{array}$ & Counts & $\begin{array}{l}\text { k-Means } \\
\text { Volume } \\
\text { Fraction }\end{array}$ \\
\hline LD C-S-H & 0.0190 & -0.10 & 4 & 15 & 0.224 & 0.20 & 0.16 & 7.22 & 4.75 & 272 & 0.344 \\
\hline HD C-S-H & 0.0240 & -0.04 & 20 & 16 & 0.426 & 0.71 & 0.28 & 23.04 & 5.01 & 281 & 0.356 \\
\hline $\mathrm{CH}$ & 0.0150 & 0.47 & 33 & 18 & 0.320 & 1.40 & 0.39 & 26.99 & 8.02 & 183 & 0.232 \\
\hline $\begin{array}{c}\text { Anisotropic } \\
\text { CH }\end{array}$ & 0.0010 & -0.33 & 61 & 13 & 0.014 & 2.67 & 0.61 & 54.16 & 11.53 & 43 & 0.054 \\
\hline $\begin{array}{c}\text { Clinker } \\
\text { Interface }\end{array}$ & 0.0007 & -0.44 & 90 & 20 & 0.016 & 5.86 & 1.66 & 88.35 & 10.11 & 11 & 0.014 \\
\hline
\end{tabular}

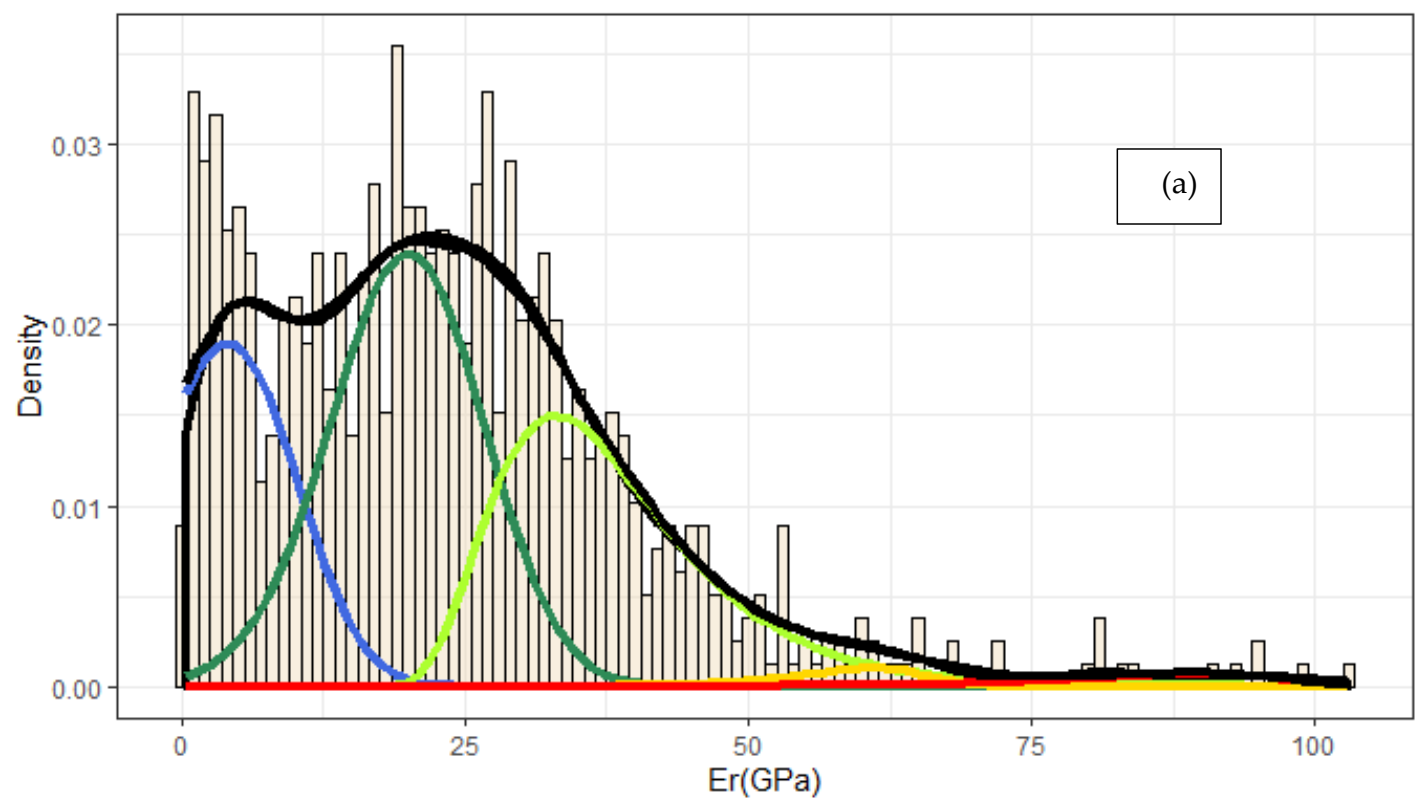

Figure 4. Cont. 


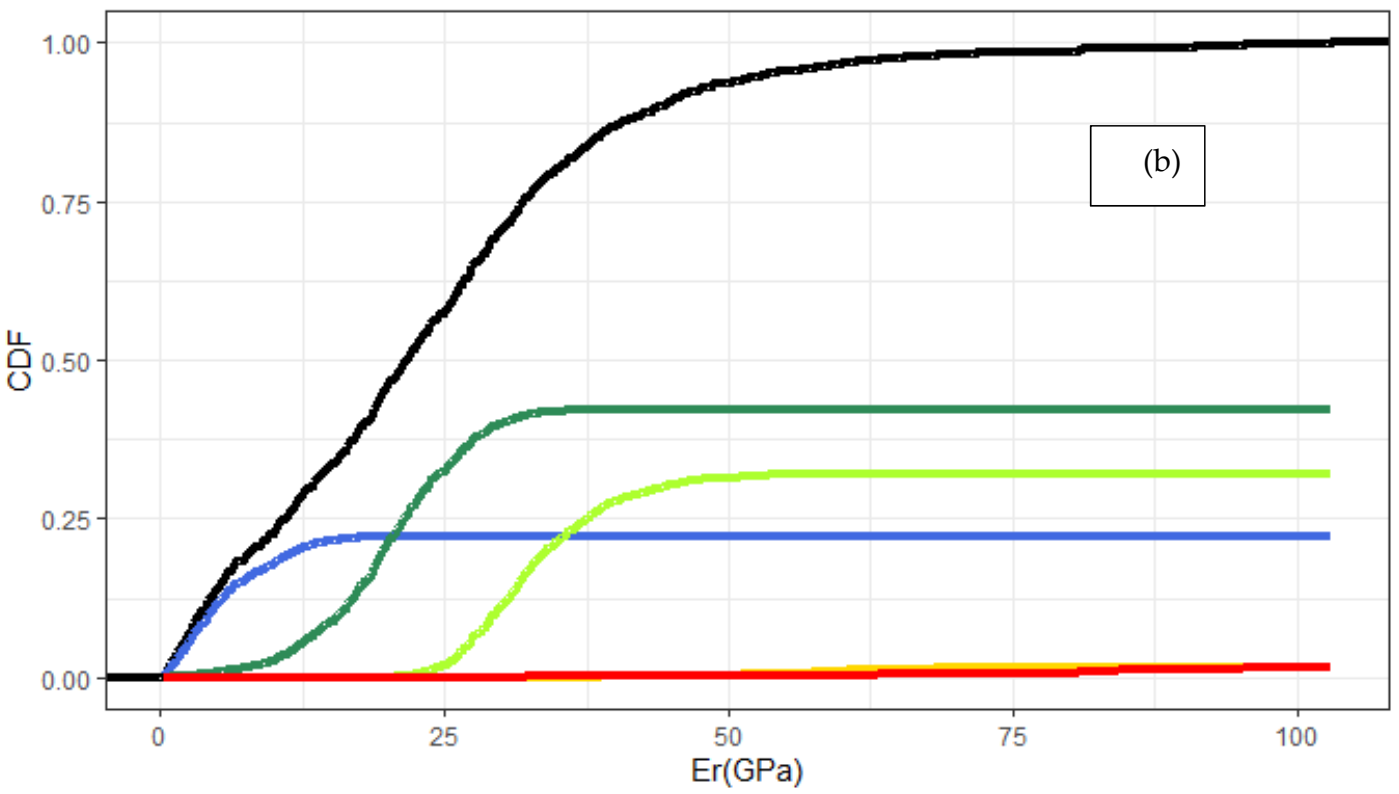

Figure 4. (a) Deconvolution of Portland Cement phases using Fraser-Suzuki function to fit nanoindentation data. The parameters are presented in Table 2. (b) the respective cumulative distribution fitting. Colors represent: blue: low density (LD) Calcium(-Silicate)-Hydrates (C-S-H.) green: high density (HD) $\mathrm{C}-\mathrm{S}-\mathrm{H}$, light green: $\mathrm{CH}$, orange: anisotropic $\mathrm{CH}$, red: clinker interface.

By using the Shiny app, which was developed within this work [62], and by performing the PDA, it is evidenced that the probabilities of Portland Cement phases deviate among individuals' judgements. Thereby, the authors recommend an alternative route than the PDF deconvolution procedure itself as a method for estimation of cement phases. The foundation is to create a database with labelled data, unbiased by the analyst, and use this source to classify new unlabeled data. The statistical summary is presented in Table 2. The mean values for $E_{r}$ are acceptable (Table 1) by both analyses; however, the variation of the mean values is minimized only in case of k-means. Also, high stiffness phases can be determined almost identically by using these two approaches. This fact may be attributed in high stiffness contrast between $\mathrm{CH}$ and clinker interface, that allows sufficient separation of data. The lower stiffness phases in cement matrix demonstrate a dense scattering of values, and the range of $E_{r}$ to describe individual phases contains data that could be attributed to a different chemical structure. Thus, it can be understood that phase identification of cement phases is not a one-dimensional problem. As a result, the correlation of chemical structure to material parameters is more reliable when using the k-means approach.

\subsection{K-Nearest Neighbors-KNN}

K-Nearest Neighbors (KNN) algorithm was utilized since it is possible to perform multiclass classification of data. It is based on the simple principle of distance calculation between data points [14], based on Euclidean distance in the present case, and recognition of classes based on similarity. The probability of a data point to be classified in a specific group of points is solely dependent on minimization of distance between the reference data points [4]. Thus, the result of KNN classification is highly related to the selection of the integer parameter " $k$ ", which accounts for the number of nearest neighbors [14]. Tuning was performed as presented in (Table A2, Figure 5), in order to minimize error value. Dispersion value accounts for the radius of the largest Euclidean ball containing no points. A graphical representation using KNN approach is presented in Figure 6. The variables importance that were involved from nanoindentation raw data demonstrate that predictions are mainly dependent on material parameters $E_{r}$ and $H$. 

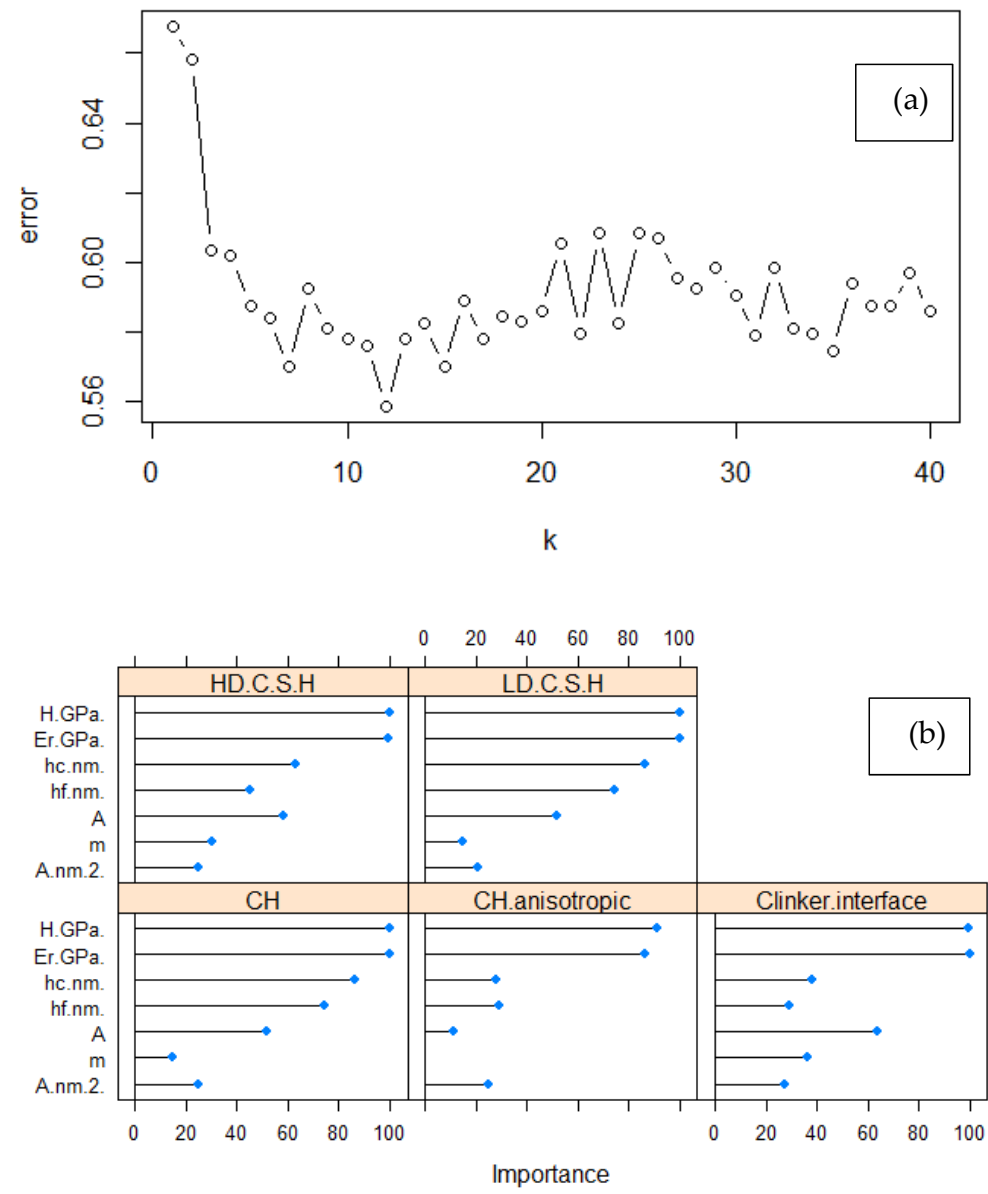

Figure 5. KNN model (a) hyperparameter tuning to identify the optimum number of $\mathrm{k}$ nearest neighbors, and (b) variables importance.

Input: k-means labeled Nanoindentation dataset spitted in $80 \%$ training and $20 \%$ test dataset

KNN model was tuned based on optimization of Accuracy metric for $k$ number of neighbors from 1 to 40 .

The best hyperparameter was identified as $\mathrm{k}=12$

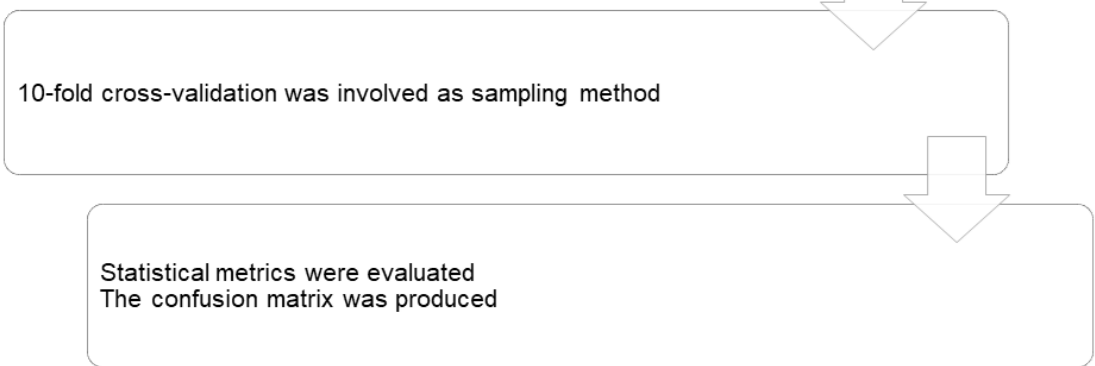

Figure 6. Graphical representation of the stepwise refinement process of K-Nearest Neighbors (KNN) classification for a sort program development.

Since the analysis is performed in the whole data entity, the majority data representation by LD C-S-H class provides sufficient information for predictive performance, resulting in an F1 score of 0.975 . However, KNN algorithm is highly affected by the imbalance in the dataset classes, as can be envisioned 
in Table 3. Although the prediction of HD C-S-H phase is acceptable, the F1 scores (maximum value is 1 for excellent prediction) for clinker interface and anisotropic $\mathrm{CH}$ phases demonstrate some drawbacks. This is also attributed to lack of data in these classes as demonstrated in the confusion matrix (Table A3), and also the imbalance in the training set deteriorates the prediction-ability. Moreover, even though micro and macro metrics overcome the random guess predictive ability of the proposed KNN model, still three classes suffer by inaccurate predictions. This is attributed to contribution from the majority of data, which are allocated in LD and HD C-S-H phases (imbalance) and increase the average of each equally weighted metric in case of micro, and in a lesser extend in macro. This is connected with total number of $T P, T N, F P, F N$. Large classes significantly affect the micro-metric values, and thus these values are closer to the metrics of LD and HD C-S-H phases.

Table 3. Statistic Metrics about KNN model.

\begin{tabular}{|c|c|c|c|c|c|}
\hline KNN Metrics & $\mathrm{CH}$ & Anisotropic $\mathrm{CH}$ & $\begin{array}{l}\text { Clinker } \\
\text { Interface }\end{array}$ & HD C-S-H & LD C-S-H \\
\hline $\begin{array}{c}\text { Support } \\
\text { (Counts-Test dataset) }\end{array}$ & 26 & 27 & 7 & 44 & 62 \\
\hline Precision & 0.500000 & 0.583333 & 0.333333 & 0.727273 & 1.000000 \\
\hline Recall & 0.730769 & 0.259259 & 0.125000 & 0.909091 & 0.951613 \\
\hline $\mathrm{F} 1$ & 0.593750 & 0.358974 & 0.181818 & 0.808081 & 0.975207 \\
\hline Accuracy & & & 0.754491 & & \\
\hline MacroAvgPrecision & & & 0.628788 & & \\
\hline MacroAvgRecall & & & 0.595147 & & \\
\hline MacroAvgF1 & & & 0.583566 & & \\
\hline MicroAvgPrecision & & & 0.754491 & & \\
\hline MicroAvgRecall & & & 0.754491 & & \\
\hline MicroAvgF1 & & & 0.754491 & & \\
\hline
\end{tabular}

\subsection{Random Forrest-RF}

Random Forest (RF) is a decision tree-based algorithm, which can perform both regression and classification [4]. It is a multifaceted algorithm that is usually used when there is uncertainty whether algorithm may be a more appropriate fit for the classification problem [4]. By the application of the algorithm, a tree is structured to correlate consecutive choices or outcomes, with branches indicating that each option is mutually exclusive [14]. RFs are sensitive to the training dataset in their predictions; thus, sampling method is important in reproducibility of results. Bagging method was adopted in present case, which maintained the same bin size of data points within each tree formation by replacement, and consequently the results are not dependent on the gradual reduction in the sampling size. Finally, data are classified based on the majority of votes by the classification tree [4]. The graphical representation of RF is summarized in Figure 7.

RF model demonstrated higher adaptivity on the training dataset and provided sufficient classification metrics. Phase identification is mainly dependent on $E_{r}$ and secondarily on $H$, while the other variables minorly affect the prediction accuracy as depicted in Figure 8. RF model lead to F1 scores that exceeded 0.909 in all occasions (Table 4), namely LD and HD C-S-H phase, CH phase and its anisotropic configuration, and the interface to clinker. The overall model accuracy exceeded $97.5 \%$, and holds promise for successful identification of unlabeled data. The weakness of the RF model is identified in Precision metric-in order to improve precision another algorithm should be used, in order to generate less false negatives during prediction in test dataset. High recall is the reason why the MacroAvgRecall is higher than MicroAvgRecall. The rest of macro- and micro-metrics follow the expected trend; therefore, macro-metrics are closer to minority classes and micro-metrics are 
closer to majority classes. No bias was observed in the confusion matrix (Table A4) in the prediction of majority class.

Input: k-means labeled Nanoindentation dataset spitted in $80 \%$ training and $20 \%$ test dataset

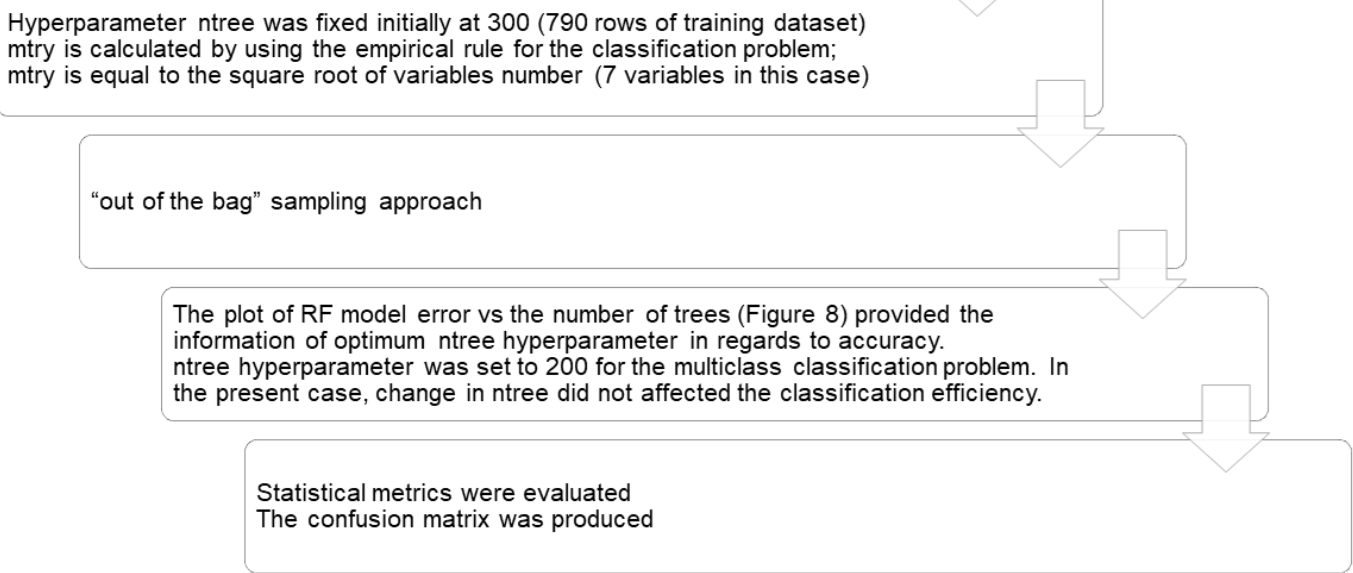

Figure 7. Graphical representation of the stepwise refinement process of Random Forest (RF) classification for a sort program development.

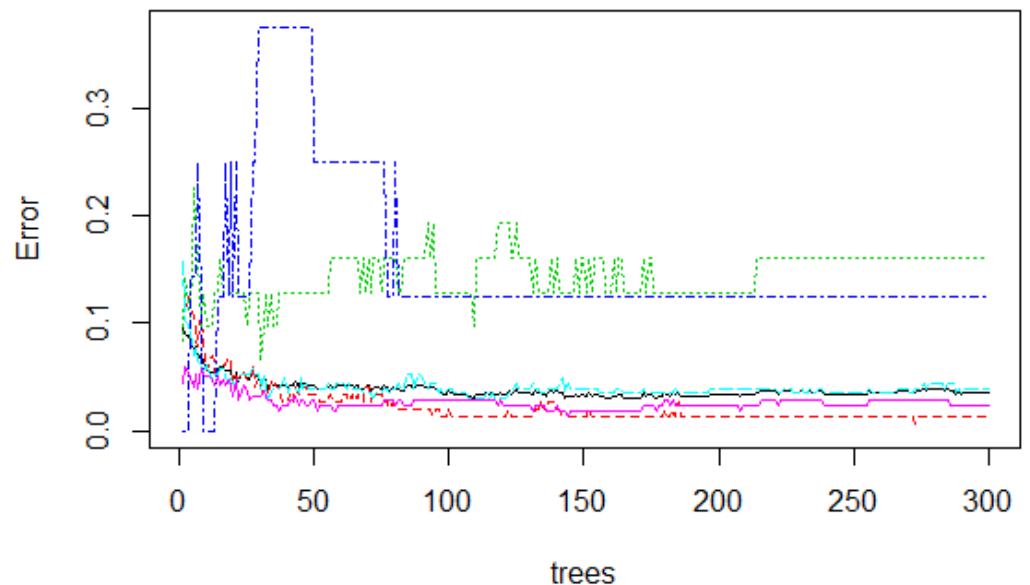

(a)
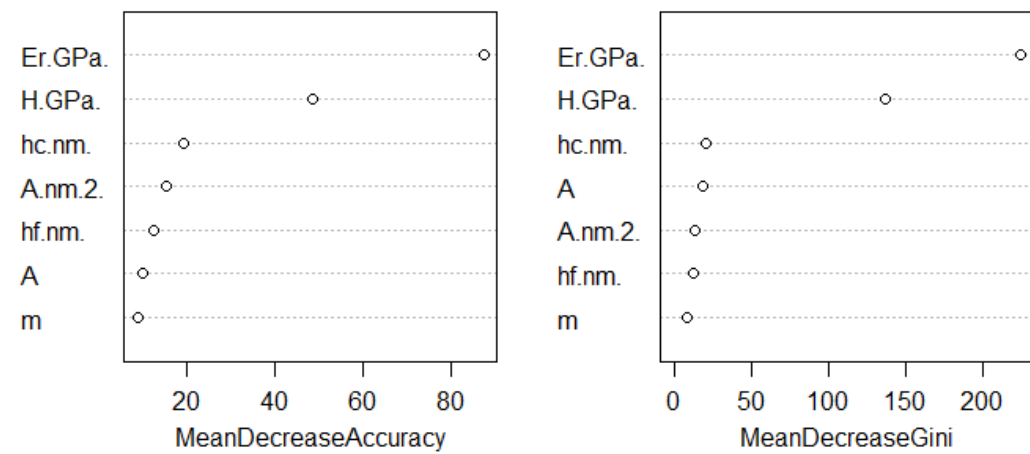

(b)

Figure 8. Random Forest (RF) model: (a) error plot vs. number of trees. Colors in figure account for; red is the LD C-S-H phase, magenta is the HD C-S-H phase, with light blue is the CH phase, with green is the anisotropic $\mathrm{CH}$ phase, and blue is the clinker interface, and (b) variables importance. 
Table 4. Statistic Metrics about Random Forest model.

\begin{tabular}{cccccc}
\hline RF Metrics & CH & Anisotropic CH & $\begin{array}{c}\text { Clinker } \\
\text { Interface }\end{array}$ & HD C-S-H & LD C-S-H \\
\hline $\begin{array}{c}\text { Support } \\
\text { (Counts-Test dataset) }\end{array}$ & 42 & 10 & 3 & 53 & 59 \\
\hline Precision & 1.000000 & 0.833333 & 1.000000 & 0.963636 & 1.000000 \\
\hline Recall & 0.904762 & 1.000000 & 1.000000 & 1.000000 & 1.000000 \\
\hline F1 & 0.950000 & 0.909091 & 1.000000 & 0.981482 & 1.000000 \\
\hline Accuracy & & 0.976048 & \\
\hline MacroAvgPrecision & & 0.959394 & \\
\hline MacroAvgRecall & & 0.980952 & \\
\hline MacroAvgF1 & & 0.968115 & \\
\hline MicroAvgPrecision & & 0.976048 & \\
\hline MicroAvgRecall & & 0.976048 & \\
\hline MicroAvgF1 & 0.976048 \\
\hline
\end{tabular}

\subsection{Support Vector Machine-SVM}

Support Vector Machine (SVM) is based on statistical learning theory and consists of heuristic algorithms [4]. Multi-dimensional data are fitted using a kernel function to provide an analogous representation, which simplifies the classification process. The kernel suitability is determined by the similarity of the kernel function to the representation of data in a high-dimensional space [14]. The hyperplane function is the boundary that separates the data for classification [8]. The implementation of SVM is summarized in Figure 9.

Input: k-means labeled Nanoindentation dataset spitted in $80 \%$

training and $20 \%$ test dataset

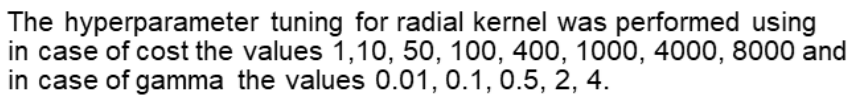

The adopted sampling method is 10 -fold cross-validation deriving as the best parameters (cost, gamma) $=(4000,0.01)$. The detailed performance results are summarized

Bound-constraint SVC was performed using Gaussian and ANOVA kernels in order to improve classification metrics.

Hyperparameters of cost and degree were set at 100 and 1, respectively, for both Gaussian and ANOVA kernel.

Figure 9. Graphical representation of the stepwise refinement process of Support Vector Machine (SVM) classification for a sort program development.

Support Vector Machine classification was first applied using a simple radial kernel function (Table 5). Hyperparameter tuning (Table A5) was performed to find optimum values for kernel complexity by cost hyperparameter adjustment. Similarly, optimum gamma value was selected, which is correlated to the retention of "bad" data points during training of the model (the higher the gamma 
value, the "bad" values are disregarded). However, even after tuning, the precision in anisotropic $\mathrm{CH}$ still suffered, and thus a lowered F1 was observed comparatively to other phases. Consequently, another kernel function should be considered to separate effectively the data into correct classes.

Table 5. Statistic Metrics about Radial Support Vector Classification (SVC) model.

\begin{tabular}{cccccc}
\hline $\begin{array}{c}\text { SVC Radial Kernel } \\
\text { Classification } \\
\text { Metrics }\end{array}$ & CH & Anisotropic CH & $\begin{array}{c}\text { Clinker } \\
\text { Interface }\end{array}$ & HD C-S-H & LD C-S-H \\
\hline $\begin{array}{c}\text { Support } \\
\text { (Counts-Test dataset) }\end{array}$ & 39 & 11 & 3 & 56 & 58 \\
\hline Precision & 0.973684 & 0.833333 & 1.000000 & 1.000000 & 0.983051 \\
\hline Recall & 0.948718 & 0.909091 & 1.000000 & 0.982143 & 1.000000 \\
\hline F1 & 0.961039 & 0.869565 & 1.000000 & 0.990991 & 0.991453 \\
\hline Accuracy & & 0.976048 & & \\
\hline MacroAvgPrecision & & 0.958014 & & \\
\hline MacroAvgRecall & & 0.967990 & & \\
\hline MacroAvgF1 & & 0.962610 & \\
\hline MicroAvgPrecision & & 0.976048 & \\
\hline MicroAvgRecall & & 0.976048 & \\
\hline MicroAvgF1 & 0.976048 & \\
\hline
\end{tabular}

To handle prediction weakness of Anisotropic CH class, Gaussian kernel function was considered (Table 6). After several trials, the optimum values of cost and gamma were identified as 100 and 1 , respectively. The kernel function change was effective, as Recall metric was higher compared to radial kernel, but no effect was evidenced in Precision. Thus, F1 score demonstrated an increment. The higher prediction efficiency of Anisotropic $\mathrm{CH}$ class was accompanied with reduction by 1 and $2 \%$ in the F1 score of LD and HD C-S-H phase, respectively; still, F1 score is considerably high. Macroand micro-averaged metrics demonstrated the same trend as in case of radial kernel SVC.

Table 6. Statistic Metrics about Gaussian SVC model.

\begin{tabular}{cccccc}
\hline $\begin{array}{c}\text { SVC Gaussian Kernel } \\
\text { Classification Metrics }\end{array}$ & CH & Anisotropic CH & $\begin{array}{c}\text { Clinker } \\
\text { Interface }\end{array}$ & HD C-S-H & LD C-S-H \\
\hline Support (Counts-Test dataset) & 41 & 10 & 3 & 56 & 57 \\
\hline Precision & 1.000000 & 0.833333 & 1.000000 & 0.981818 & 0.966102 \\
\hline Recall & 0.926829 & 1.000000 & 1.000000 & 0.964286 & 1.000000 \\
\hline F1 & 0.962025 & 0.909091 & 1.000000 & 0.972973 & 0.982759 \\
\hline Accuracy & & 0.970060 & & \\
\hline MacroAvgPrecision & & 0.956251 & & \\
\hline MacroAvgRecall & & 0.978223 & \\
\hline MacroAvgF1 & & 0.965370 & \\
\hline MicroAvgPrecision & & 0.970060 & \\
\hline MicroAvgRecall & & 0.970060 & \\
\hline MicroAvgF1 & & 0.970060 & \\
\hline
\end{tabular}

In order to find out if another kernel function could overcome the prediction efficiency of Gaussian kernel, and also RF performance, ANOVA kernel function was considered (Table 7). Separability of data further improved the F1 score, which overcame 95\% in all categories, and F1 score was improved by $1 \%$ for $\mathrm{CH}$ class, and by $4.5 \%$ for anisotropic $\mathrm{CH}$. This improvement in case of Anisotropic $\mathrm{CH}$ class was influenced by Precision increment by using ANOVA kernel. Thus, even though the overall model accuracy is the same for all RF, radial SVC kernel and ANOVA SVC kernel, ANOVA approach 
could be more sensitive in the correct prediction of an unlabeled nanoindentation event (Table A6, Table A7, Table A8). Consequently, it is considered as a model that is unbiased by the data imbalance and favors dealing effectively with Portland Cement phases classification problem by achieving higher individual precision compared to the aforementioned models. This conclusion is further supported by the higher observed values of micro-metrics, with MicroAvgPrecision exceeding MacroAvgPrecision, and by achieving a very similar F1 value for both metrics.

Table 7. Statistic Metrics about ANOVA SVC model.

\begin{tabular}{|c|c|c|c|c|c|}
\hline $\begin{array}{l}\text { SVC ANOVA Kernel } \\
\text { Classification Metrics }\end{array}$ & $\mathrm{CH}$ & Anisotropic $\mathrm{CH}$ & $\begin{array}{l}\text { Clinker } \\
\text { Interface }\end{array}$ & HD C-S-H & LD C-S-H \\
\hline Support (Counts-Test dataset) & 40 & 11 & 3 & 56 & 57 \\
\hline Precision & 1.000000 & 0.916667 & 1.000000 & 0.981818 & 0.966102 \\
\hline Recall & 0.950000 & 1.000000 & 1.000000 & 0.964286 & 1.000000 \\
\hline $\mathrm{F} 1$ & 0.974359 & 0.956522 & 1.000000 & 0.972973 & 0.982759 \\
\hline Accuracy & & & 0.976048 & & \\
\hline MacroAvgPrecision & & & 0.972917 & & \\
\hline MacroAvgRecall & & & 0.982857 & & \\
\hline MacroAvgF1 & & & 0.977323 & & \\
\hline MicroAvgPrecision & & & 0.976048 & & \\
\hline MicroAvgRecall & & & 0.976048 & & \\
\hline MicroAvgF1 & & & 0.976048 & & \\
\hline
\end{tabular}

\section{Discussion}

Till now, the most common approach when studying the cement hydrated phases with nanoindentation included Probability Distribution Analysis. This approach was based solely on deconvolution by Gaussian fittings, which suffers from the non-uniqueness of the solution. The number of peaks in the density plot, and thus the number of Gaussians, create a multidimensional space when measuring the parameters of the solution, and this approach suffers by the existence of more than one global optima. As a consequence, the analysis of the same nanoindentation data may vary amongst individuals. Moreover, third and fourth statistical moments are nullified by assuming zero skewness and using Gaussians to fit data, which introduces another factor for error evolution. Within this study, a practical approach in PDA is summarized by introducing skewness by the incorporation of Fraser-Suzuki equation for fitting. The input parameters of $p_{i}, s_{i}, E_{m, I}, d_{i}$ are useful for later analysis with integrals and calculation of the composition percentage of each individual phase.

Machine Learning came up as a more efficient route to deal with the multivariate problem of nanoindentation raw data. Implementation of an unsupervised ML algorithm for unbiased determination of cement phases was demonstrated using $\mathrm{k}$-means clustering. Unsupervised phase identification showed the magnitude of variation in microstructures volume fraction. This is an introductory step for labelling the test data. Labelling is useful to perform quick evaluation of cement composition by training supervised ML models for performing quality control of the synthesized or nano-enforced cementitious structures. This approach known as semi-supervised Machine Learning is also unbiased, can be reproduced, and combines multidimensional features. Inclusion of seven variables enables establishing structure (cement phase)—property (parameters: $E_{r}, H$ ) relations to contribute in reinforcement identification due to possible enhanced propagation of hydration and nucleation aided by nanomaterials. This reinforcement is envisioned especially in cement interface (or matrix) and the compositional changes in LD, HD C-S-H, and $\mathrm{CH}$ phases, considering that better interfacial properties will improve overall performance.

Cement phase identification, which was implemented using two methodologies, a PDF fitting with skewness and unsupervised ML algorithm of k-means enabled to identify the strengths of each approach directly. In the first case, PDF was applied based on Fraser-Suzuki function in order to 
improve fitting results as asymmetry is enabled. This implementation is also available in the shape of Shiny app to fit $E_{r}$ nanoindentation data using $\mathrm{R}$ language [62]. Two significant aspects should be pointed out. Firstly, the presented case required 5 PDF fittings, which means that a 20-dimensional space (5 PDFs $\times 4$ parameters) is created for the problem solution. Thus, the proposed fitting falls within one of the local optima set of solutions. As a result, it can be understood that another solution may be chosen by another individual. Secondly, the solution in PDA is about the single-parameter problem (here: reduced elastic modulus). The deviation is high, as expected, compared to k-means clustering approach, which incorporated more variables to provide the microstructure clusters. As demonstrated in Figure 10a, the PDA predictions were biased to HD C-S-H and CH phases. This deviation was not encountered for the clinker interface, as both methodologies lead to similar results in case of $E_{r}$ value, possibly due to the low population of available data. In this direction, k-means correlated material parameters to cement chemical structure using both parameters $H$ and $E_{r}$ as input. Consequently, k-means was used for unbiased labelling of the nanoindentation data in order to feed the data for multiclass classification and perform semi-supervised Machine Learning. In conclusion, identification of cement phases using nanoindentation data is improved when it is approached as a multivariate problem with Machine Learning, which was expected in principle [45].

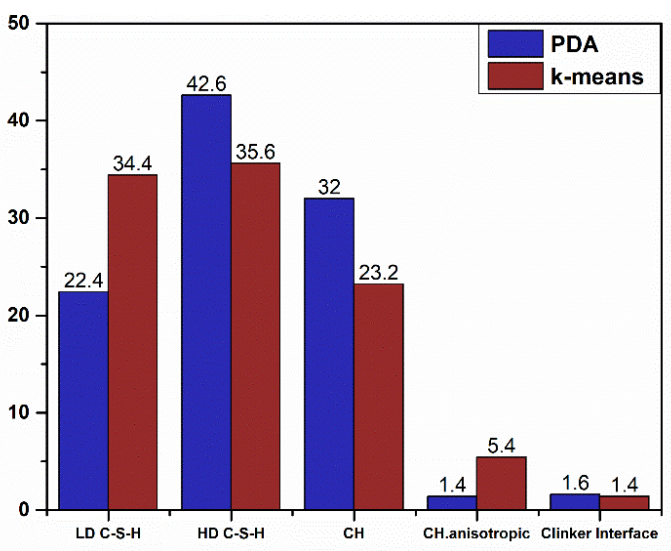

(a)

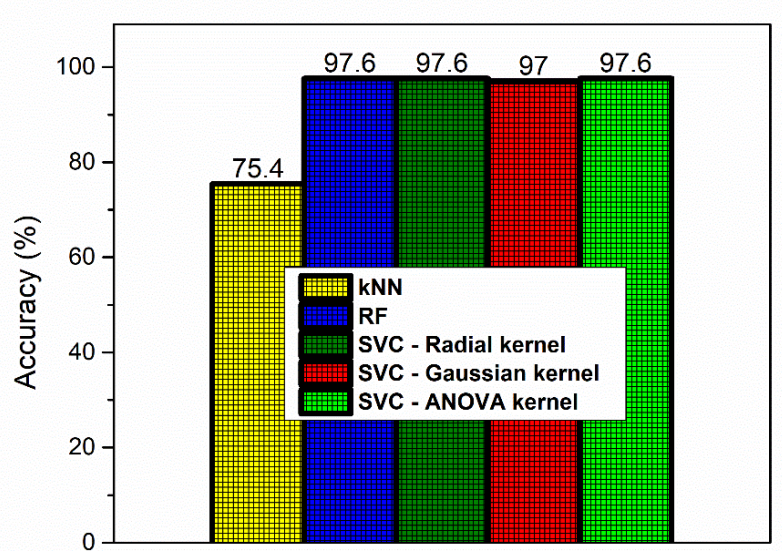

(b)

Figure 10. Summary of major results: (a) volume fraction (\%) as measured by PDA vs. k-means, (b) represent the comparative histograms of Precision, Recall, and F1-score, respectively.

In another case, $\mathrm{k}$-means clustering has been used in order to predict the nanoindentation response of a given location in FCC single crystals [51], with overall accuracy to reach $90 \%$. In this work, multiclass classification was performed using common algorithms of KNN, RF, and SVC reaching a maximum accuracy of $97.6 \%$ (Figure $10 \mathrm{~b}$ ). In detail, KNN model was trained in order to predict the classes in the test dataset. However, the model performance was not adequate even after tuning of hyperparameters, especially for the high-stiffness phases identification, reaching a minimum F1 score of 0.18 (Figure 10e). This result could be possibly correlated to the low population of data for these cement phases and imbalance in the population amongst cement microstructure classes [53]. On the other hand, all other algorithms (RF and Radial, Gaussian, and ANOVA SVC kernel types) after being properly tuned were able to use all seven variables to correctly classify nanoindentation events to Portland Cement phases. A minimum score of F1 $=0.87$ in case of Radial SVC kernel was achieved and the highest minimum-class predictive score of $\mathrm{F} 1=0.96$ was accomplished with ANOVA kernel. Also, with ANOVA kernel the weakness of SVC in Precision metric was overcome. Although RF demonstrated the same number of misclassifications in the test dataset as ANOVA SVC kernel, in fact, misclassifications are not accumulated in a single category when using ANOVA, which is preferable compared to Random Forrest. The key in finding the best possible algorithm for each scenario is identified in testing a variety of classification algorithms in order to find the right balance between 
Precision and Recall metrics, since often it is challenging to keep both high in value (Figure 11a-c). Even if Random Forrest provided the highest Recall in HD C-S-H and CH phases, it was preferred to sacrifice Recall in favor of achieving higher Precision in classification of those two phases, in order to reduce misclassification error of the trained models in future input of unseen data and achieve high prediction metrics in all hydrated cement phases.

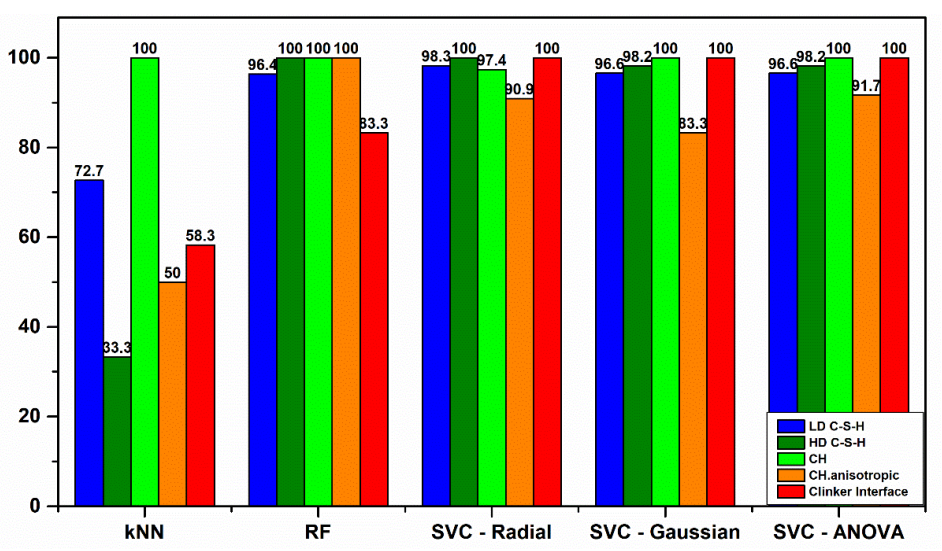

(a)

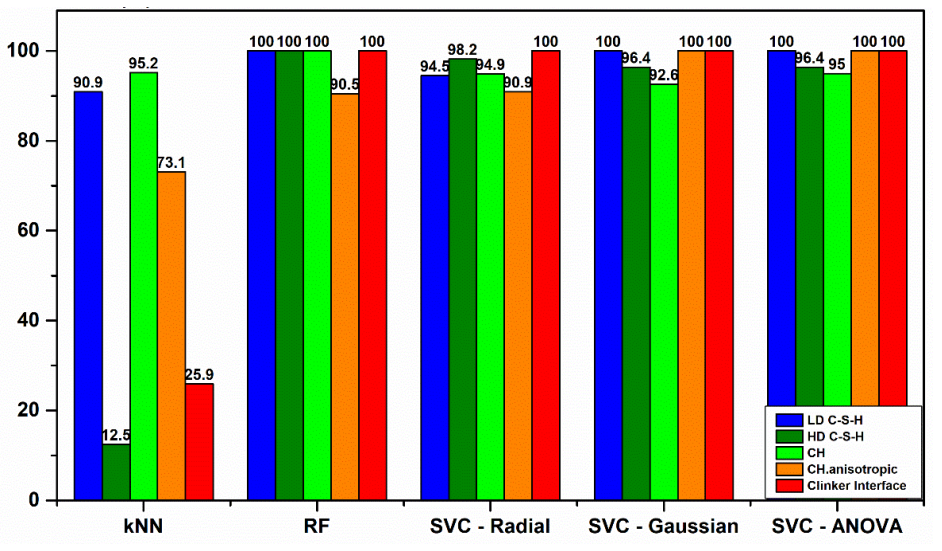

(b)

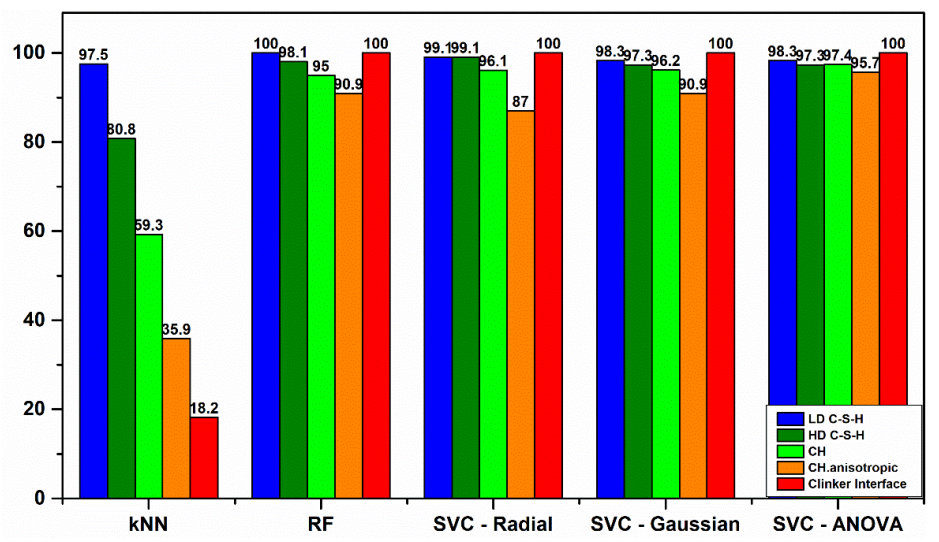

(c)

Figure 11. Summary of major results: (a-c) represent the comparative histograms of Precision, Recall, and F1-score, respectively. 


\section{Conclusions}

This study aimed in the implementation of an enhanced practice for analysis of cement microstructure with nanoindentation testing. Step-by-step methodology of data preprocessing, labelling, and classification is summarized in order to enhance interlaboratory reproducibility of the results. It is important to note that nanoindentation protocol for mapping majorly affects the usability of data for phase identification and highlights the necessity for establishing good practices in testing cement formulations. A common approach in characterization protocols is essential to enable data exchange and further developments in characterization methods. Semi-supervised Machine Learning was implemented due to the enhanced efficiency in predictive modeling of microstructure. In principle, Machine Learning exceeds traditional statistics for predictive modeling. This is derived by the inclusion of more variables, and thus data, to pattern relationships in the labeled data. The fitted patterns become more complex and contain more information for the microstructure classes. This is a gain compared to traditional statistics for prediction of cement phases, which in case of Probability Distribution Analysis, uses a single parameter for identification. Increased complexity in relations of nanoindentation data and cement microstructures enhances the level of prediction accuracy when testing other formulations not previously used for training Machine Learning models. High values obtained for prediction metrics demonstrate the transfer learning potential, which is performed with extrapolation in traditional statistic and usually suffers from poor accuracy.

This work contributes to the field of cement nanocomposites design and quality control associated with identifying the effect of low dosages of engineered nanomaterials inclusion in reinforcement assessment; microstructure, and mechanical properties of cement-based composites can also be correlated to the fabrication, workability, and hydration in optimization tasks. The extensive use of statistics in the microstructure identification in the past decades was reasonable since computational strength was limited. However, technology evolution increases the necessity of materials scientists to adapt and improve their tools and data capacity for closing the gap of new ideas for design and applicability evaluation with less effort and need for resources. In this direction, Artificial Intelligence can provide a module for enabling fast, in-line, and real-time metrological characterization of nanoindentation data. Emphasis is located on classification of newly characterized data (specimen testing) based on a labelled database, which is promising to minimize the requirement for human effort in quality control and life assessment of Portland Cement formulations. The proposed microstructure analysis of Portland Cement using AI on nanoindentation data processing provided considerable proceedings; namely, classification reached an ultimate plateau value of $97.6 \%$ model accuracy using ANOVA SVC kernel and minimum F1 score of 95\% in the five-class classification problem. Additionally, all approaches required a few seconds of computational time for clustering, training, and fitting. The high levels of accuracy hold promise for transfer learning potential and scalability of this methodology to expand prior obtained knowledge on new data.

Author Contributions: Conceptualization, E.P.K. and G.K.; methodology, E.P.K. and G.K.; software, G.K.; validation E.P.K. and G.K.; formal analysis, E.P.K. and G.K.; investigation, E.P.K. and G.K.; resources, C.A.C. and E.P.K.; data Curation, E.P.K. and G.K.; writing-original draft preparation, C.A.C., E.P.K. and G.K.; writing-review and editing, C.A.C., E.P.K. and G.K.; visualization, C.A.C., E.P.K. and G.K.; supervision, C.A.C., E.P.K. and G.K.; project administration, C.A.C. and E.P.K.; funding acquisition, C.A.C. and E.P.K. All authors have read and agreed to the published version of the manuscript.

Funding: The authors would like to acknowledge the financial support for this study partially from the European Union's Horizon 2020 research and innovation program under grant agreement No685445 (LORCENIS) and partially from the EU H2020 Project “Modified Cost Effective Fiber Based Structures With Improved Multi-Functionality And Performance" (MODCOMP) Under Grant Agreement No. 685844.

Conflicts of Interest: The authors declare no conflict of interest. 


\section{Appendix A}

Table A1. R session info and Packages (accessed date: 2019-09-30).

\begin{tabular}{|c|c|c|c|c|c|c|c|c|c|}
\hline Package & Version & Sour & ce & & Package & Version & Sour & ce & \\
\hline abind & $1.4-5$ & CRAN & $(\mathrm{R}$ & 3.6.0) & pillar & 1.4 .1 & CRAN & $(\mathrm{R}$ & 3.6.0) \\
\hline assertthat & 0.2 .1 & CRAN & (R & 3.6.0) & pkgbuild & 1.0 .3 & CRAN & (R & 3.6.0) \\
\hline backports & 1.1 .4 & CRAN & (R & 3.6.0) & pkgconfig & 2.0 .2 & CRAN & (R & 3.6.0) \\
\hline broom & 0.5 .2 & CRAN & (R & 3.6.0) & pkgload & 1.0 .2 & CRAN & (R & 3.6.0) \\
\hline callr & 3.2 .0 & CRAN & $(\mathrm{R}$ & 3.6.0) & plyr & 1.8 .4 & CRAN & $(\mathrm{R}$ & 3.6.0) \\
\hline caret & $6.0-84$ & CRAN & (R & 3.6.1) & prettyunits & 1.0 .2 & CRAN & (R & 3.6.0) \\
\hline cellranger & 1.1 .0 & CRAN & (R & 3.6.0) & pROC & 1.15 .3 & CRAN & (R & 3.6.1) \\
\hline class & $7.3-15$ & CRAN & $(\mathrm{R}$ & 3.6.1) & processx & 3.3.1 & CRAN & $(\mathrm{R}$ & 3.6.0) \\
\hline data.table & 1.12 .2 & CRAN & $(\mathrm{R}$ & 3.6.0) & prodlim & 2018.04 .18 & CRAN & $(\mathrm{R}$ & 3.6.1) \\
\hline desc & 1.2 .0 & CRAN & (R & 3.6.0) & ps & 1.3 .0 & CRAN & $(\mathrm{R}$ & 3.6.0) \\
\hline devtools & 2.2 .0 & CRAN & (R & 3.6.1) & purrr & 0.3 .2 & CRAN & (R & 3.6.0) \\
\hline digest & 0.6 .20 & CRAN & (R & 3.6.1) & R6 & 2.4 .0 & CRAN & (R & 3.6.0) \\
\hline dplyr & 0.8 .3 & CRAN & $(\mathrm{R}$ & 3.6.1) & randomForest & 4.6-14 & CRAN & $(\mathrm{R}$ & 3.6.1) \\
\hline DT & 0.9 & CRAN & (R & 3.6.0) & Rcpp & 1.0 .1 & CRAN & (R & 3.6.0) \\
\hline e1071 & $1.7-2$ & CRAN & $(\mathrm{R}$ & 3.6.1) & readr & 1.3.1 & CRAN & $(\mathrm{R}$ & 3.6.0) \\
\hline ellipsis & 0.2 .0 .1 & CRAN & $(\mathrm{R}$ & 3.6.1) & readxl & 1.3 .1 & CRAN & $(\mathrm{R}$ & 3.6.1) \\
\hline ggplot2 & 3.1 .1 & CRAN & $(\mathrm{R}$ & 3.6.0) & reshape2 & 1.4 .3 & CRAN & $(\mathrm{R}$ & 3.6.0) \\
\hline glue & 1.3.1 & CRAN & $(\mathrm{R}$ & 3.6.0) & rJava & $0.9-11$ & CRAN & $(\mathrm{R}$ & 3.6.0) \\
\hline htmltools & 0.3 .6 & CRAN & $(\mathrm{R}$ & 3.6.0) & rlang & 0.4 .0 & CRAN & $(\mathrm{R}$ & 3.6.1) \\
\hline htmlwidgets & 1.3 & CRAN & (R & 3.6.0) & scales & 1.0 .0 & CRAN & $(\mathrm{R}$ & 3.6.0) \\
\hline httr & 1.4 .0 & CRAN & $(\mathrm{R}$ & 3.6.0) & segmented & $1.0-0$ & CRAN & $(\mathrm{R}$ & 3.6.0) \\
\hline kernlab & $0.9-27$ & CRAN & (R & 3.6.0) & sessioninfo & 1.1.1 & CRAN & (R & 3.6.0) \\
\hline labeling & 0.3 & CRAN & (R & $3.6 .0)$ & shiny & 1.3 .2 .9001 & Gith & $\mathrm{ub}$ & (rstudio/shiny@89bd7e9) \\
\hline lattice & $0.20-38$ & CRAN & (R & $3.6 .0)$ & sjlabelled & 1.1.1 & CRAN & (R & 3.6.1) \\
\hline lava & 1.6 .6 & CRAN & (R & $3.6 .1)$ & stringi & 1.4 .3 & CRAN & (R & $3.6 .0)$ \\
\hline lazyeval & 0.2 .2 & CRAN & $(\mathrm{R}$ & 3.6.0) & stringr & 1.4 .0 & CRAN & $(\mathrm{R}$ & 3.6.0) \\
\hline lubridate & 1.7 .4 & CRAN & (R & $3.6 .0)$ & survival & $2.44-1.1$ & CRAN & (R & $3.6 .0)$ \\
\hline magic & $1.5-9$ & CRAN & (R & $3.6 .0)$ & testthat & 2.2 .1 & CRAN & (R & 3.6.1) \\
\hline magrittr & 1.5 & CRAN & (R & 3.6.0) & tibble & 2.1 .3 & CRAN & (R & 3.6.0) \\
\hline MASS & 7.3-51.4 & CRAN & $(\mathrm{R}$ & 3.6.0) & tidyr & 0.8 .3 & CRAN & $(\mathrm{R}$ & 3.6.0) \\
\hline Matrix & $1.2-17$ & CRAN & (R & 3.6.0) & tidyselect & 0.2 .5 & CRAN & (R & 3.6.0) \\
\hline mclust & 5.4 .5 & CRAN & (R & 3.6.1) & tidyverse & 1.2 .1 & CRAN & (R & 3.6.0) \\
\hline mixtools & 1.1 .0 & CRAN & (R & 3.6.1) & timeDate & 3043.102 & CRAN & (R & $3.6 .0)$ \\
\hline mlbench & $2.1-1$ & CRAN & (R & 3.6.1) & usethis & 1.5 .0 & CRAN & (R & 3.6.0) \\
\hline MLmetrics & 1.1 .1 & CRAN & (R & 3.6.1) & withr & 2.1 .2 & CRAN & (R & 3.6.0) \\
\hline ModelMetrics & 1.2 .2 & CRAN & $(\mathrm{R}$ & 3.6.1) & xlsx & 0.6 .1 & CRAN & $(\mathrm{R}$ & 3.6.0) \\
\hline modelr & 0.1 .4 & CRAN & (R & 3.6.0) & xlsxjars & 0.6 .1 & CRAN & (R & 3.6.0) \\
\hline pdfCluster & $1.0-3$ & CRAN & (R & 3.6.1) & $\mathrm{xml2}$ & 1.2 .0 & CRAN & (R & 3.6.0) \\
\hline
\end{tabular}

Table A2. Detailed performance results of KNN hyperparameter tuning.

\begin{tabular}{ccccccccc}
\hline $\mathbf{k}$ & Error & Dispersion & $\mathbf{k}$ & Error & dispersion & $\mathbf{k}$ & Error & Dispersion \\
\hline $\mathbf{1}$ & 0.667742 & 0.047836 & $\mathbf{1 5}$ & 0.569765 & 0.042177 & $\mathbf{2 8}$ & 0.592217 & 0.058813 \\
\hline $\mathbf{2}$ & 0.658013 & 0.048698 & $\mathbf{1 6}$ & 0.589094 & 0.050706 & $\mathbf{2 9}$ & 0.598566 & 0.062328 \\
\hline $\mathbf{3}$ & 0.603405 & 0.083304 & $\mathbf{1 7}$ & 0.57788 & 0.04982 & $\mathbf{3 0}$ & 0.590502 & 0.071531 \\
\hline $\mathbf{4}$ & 0.601843 & 0.073970 & $\mathbf{1 8}$ & 0.584485 & 0.053367 & $\mathbf{3 1}$ & 0.579186 & 0.080118 \\
\hline $\mathbf{5}$ & 0.587276 & 0.075722 & $\mathbf{1 9}$ & 0.582693 & 0.058426 & $\mathbf{3 2}$ & 0.598464 & 0.068451 \\
\hline $\mathbf{6}$ & 0.584101 & 0.054559 & $\mathbf{2 0}$ & 0.586022 & 0.055453 & $\mathbf{3 3}$ & 0.580901 & 0.065523 \\
\hline
\end{tabular}


Table A2. Cont.

\begin{tabular}{ccccccccc}
\hline $\mathbf{k}$ & Error & Dispersion & $\mathbf{k}$ & Error & dispersion & $\mathbf{k}$ & Error & Dispersion \\
\hline $\mathbf{7}$ & 0.569739 & 0.063925 & $\mathbf{2 1}$ & 0.605146 & 0.048521 & $\mathbf{3 4}$ & 0.57934 & 0.067627 \\
\hline $\mathbf{8}$ & 0.592217 & 0.057005 & $\mathbf{2 2}$ & 0.579519 & 0.048427 & $\mathbf{3 5}$ & 0.574552 & 0.065912 \\
\hline $\mathbf{9}$ & 0.581055 & 0.071392 & $\mathbf{2 3}$ & 0.608321 & 0.053482 & $\mathbf{3 6}$ & 0.593907 & 0.054005 \\
\hline $\mathbf{1 0}$ & 0.577829 & 0.059211 & $\mathbf{2 4}$ & 0.582617 & 0.040259 & $\mathbf{3 7}$ & 0.58743 & 0.066458 \\
\hline $\mathbf{1 1}$ & 0.576114 & 0.043615 & $\mathbf{2 5}$ & 0.608244 & 0.047015 & $\mathbf{3 8}$ & 0.587353 & 0.068217 \\
\hline $\mathbf{1 2}$ & 0.558577 & 0.045023 & $\mathbf{2 6}$ & 0.606682 & 0.044701 & $\mathbf{3 9}$ & 0.59703 & 0.060446 \\
\hline $\mathbf{1 3}$ & 0.577803 & 0.026752 & $\mathbf{2 7}$ & 0.595366 & 0.060007 & $\mathbf{4 0}$ & 0.585689 & 0.068017 \\
\hline $\mathbf{1 4}$ & 0.58254 & 0.056609 & & & & & & \\
\hline
\end{tabular}

Table A3. Confusion matrix of the testing dataset and the predicted Portland Cement Phases by KNN model.

\begin{tabular}{cccccc}
\hline KNN Classification & CH & Anisotropic CH & Clinker Interface & HD C-S-H & LD C-S-H \\
\hline CH & 19 & 1 & 0 & 6 & 0 \\
\hline Anisotropic CH & 13 & 7 & 2 & 5 & 0 \\
\hline Clinker interface & 2 & 4 & 1 & 1 & 0 \\
\hline HD C-S-H & 4 & 0 & 0 & 40 & 0 \\
\hline LD C-S-H & 0 & 0 & 0 & 3 & 59 \\
\hline
\end{tabular}

Table A4. Confusion matrix of the testing dataset and the predicted Portland Cement Phases by random forrest model.

\begin{tabular}{cccccc}
\hline RF Classification & CH & Anisotropic CH & Clinker Interface & HD C-S-H & LD C-S-H \\
\hline CH & 38 & 2 & 0 & 2 & 0 \\
\hline Anisotropic CH & 0 & 10 & 0 & 0 & 0 \\
\hline Clinker interface & 0 & 0 & 3 & 0 & 0 \\
\hline HD C-S-H & 0 & 0 & 0 & 53 & 0 \\
\hline LD C-S-H & 0 & 0 & 0 & 0 & 59 \\
\hline
\end{tabular}

Table A5. Detailed performance results of Radial Support Vector Classification (SVC) kernel hyperparameter tuning.

\begin{tabular}{c|c|c|c|c|c|c|c|c|c}
\hline $\mathbf{\#}$ & Gamma & Cost & Error & Dispersion & $\mathbf{\#}$ & Gamma & Cost & Error & Dispersion \\
\hline $\mathbf{1}$ & 0.01 & 1 & 0.121941 & 0.048335 & $\mathbf{2 1}$ & 0.01 & 400 & 0.036918 & 0.02526 \\
\hline $\mathbf{2}$ & 0.1 & 1 & 0.072248 & 0.035021 & $\mathbf{2 2}$ & 0.1 & 400 & 0.052970 & 0.036494 \\
\hline $\mathbf{3}$ & 0.5 & 1 & 0.083436 & 0.032799 & $\mathbf{2 3}$ & 0.5 & 400 & 0.083410 & 0.036922 \\
\hline $\mathbf{4}$ & $\mathbf{2}$ & 1 & 0.125166 & 0.049255 & $\mathbf{2 4}$ & 2 & 400 & 0.104327 & 0.047335 \\
\hline $\mathbf{5}$ & 4 & 1 & 0.146032 & 0.042723 & $\mathbf{2 5}$ & 4 & 400 & 0.131618 & 0.034375 \\
\hline $\mathbf{6}$ & 0.01 & 10 & 0.051357 & 0.014704 & $\mathbf{2 6}$ & 0.01 & 1000 & 0.040143 & 0.026622 \\
\hline $\mathbf{7}$ & 0.1 & 10 & 0.052919 & 0.033045 & $\mathbf{2 7}$ & 0.1 & 1000 & 0.056144 & 0.038113 \\
\hline $\mathbf{8}$ & 0.5 & 10 & 0.086610 & 0.034644 & $\mathbf{2 8}$ & 0.5 & 1000 & 0.081797 & 0.034984 \\
\hline $\mathbf{9}$ & $\mathbf{2}$ & 10 & 0.110701 & 0.045551 & $\mathbf{2 9}$ & 2 & 1000 & 0.104327 & 0.047335 \\
\hline $\mathbf{1 0}$ & 4 & 10 & 0.134793 & 0.036901 & $\mathbf{3 0}$ & 4 & 1000 & 0.131618 & 0.034375 \\
\hline
\end{tabular}


Table A5. Cont.

\begin{tabular}{c|c|c|c|c|c|c|c|c|c}
\hline \# & Gamma & Cost & Error & Dispersion & \# & Gamma & Cost & Error & Dispersion \\
\hline $\mathbf{1 1}$ & 0.01 & 50 & 0.040118 & 0.028672 & $\mathbf{3 1}$ & 0.01 & 4000 & 0.030492 & 0.028863 \\
\hline $\mathbf{1 2}$ & 0.1 & 50 & 0.048157 & 0.026341 & $\mathbf{3 2}$ & 0.1 & 4000 & 0.049770 & 0.039092 \\
\hline $\mathbf{1 3}$ & 0.5 & 50 & 0.083436 & 0.035396 & $\mathbf{3 3}$ & 0.5 & 4000 & 0.086610 & 0.033016 \\
\hline $\mathbf{1 4}$ & $\mathbf{2}$ & 50 & 0.107501 & 0.045313 & $\mathbf{3 4}$ & 2 & 4000 & 0.104327 & 0.047335 \\
\hline $\mathbf{1 5}$ & 4 & 50 & 0.134793 & 0.036901 & $\mathbf{3 5}$ & 4 & 4000 & 0.131618 & 0.034375 \\
\hline $\mathbf{1 6}$ & 0.01 & 100 & 0.038530 & 0.024244 & $\mathbf{3 6}$ & 0.01 & 8000 & 0.033769 & 0.030845 \\
\hline $\mathbf{1 7}$ & 0.1 & 100 & 0.051382 & 0.032078 & $\mathbf{3 7}$ & 0.1 & 8000 & 0.048182 & 0.032232 \\
\hline $\mathbf{1 8}$ & 0.5 & 100 & 0.089836 & 0.033124 & $\mathbf{3 8}$ & 0.5 & 8000 & 0.086610 & 0.033016 \\
\hline $\mathbf{1 9}$ & $\mathbf{2}$ & 100 & 0.102739 & 0.048516 & $\mathbf{3 9}$ & 2 & 8000 & 0.104327 & 0.047335 \\
\hline $\mathbf{2 0}$ & 4 & 100 & 0.136380 & 0.034517 & $\mathbf{4 0}$ & 4 & 8000 & 0.131618 & 0.034375 \\
\hline
\end{tabular}

Table A6. Confusion matrix of the testing dataset and the predicted Portland Cement Phases by Radial SVC model.

\begin{tabular}{cccccc}
\hline $\begin{array}{c}\text { SVC Radial Kernel } \\
\text { Classification }\end{array}$ & CH & Anisotropic CH & Clinker Interface & HD C-S-H & LD C-S-H \\
\hline CH & 37 & 2 & 0 & 0 & 0 \\
\hline Anisotropic CH & 1 & 10 & 0 & 0 & 0 \\
\hline Clinker interface & 0 & 0 & 3 & 0 & 0 \\
\hline HD C-S-H & 0 & 0 & 0 & 55 & 1 \\
\hline LD C-S-H & 0 & 0 & 0 & 0 & 58 \\
\hline
\end{tabular}

Table A7. Confusion matrix of the testing dataset and the predicted Portland Cement Phases by Gaussian SVC model.

\begin{tabular}{cccccc}
\hline $\begin{array}{c}\text { SVC Gaussian } \\
\text { Kernel } \\
\text { Classification }\end{array}$ & CH & Anisotropic CH & Clinker Interface & HD C-S-H & LD C-S-H \\
\hline CH & 38 & 2 & 0 & 1 & 0 \\
\hline Anisotropic CH & 0 & 10 & 0 & 0 & 0 \\
\hline Clinker interface & 0 & 0 & 3 & 0 & 0 \\
\hline HD C-S-H & 0 & 0 & 0 & 54 & 2 \\
\hline LD C-S-H & 0 & 0 & 0 & 0 & 57 \\
\hline
\end{tabular}

Table A8. Confusion matrix of the testing dataset and the predicted Portland Cement Phases by ANOVA SVC model.

\begin{tabular}{cccccc}
\hline $\begin{array}{c}\text { SVC ANOVA } \\
\text { Kernel } \\
\text { Classification }\end{array}$ & CH & Anisotropic CH & Clinker Interface & HD C-S-H & LD C-S-H \\
\hline CH & 38 & 1 & 0 & 1 & 0 \\
\hline Anisotropic CH & 0 & 11 & 0 & 0 & 0 \\
\hline Clinker interface & 0 & 0 & 3 & 0 & 0 \\
\hline HD C-S-H & 0 & 0 & 0 & 54 & 2 \\
\hline LD C-S-H & 0 & 0 & 0 & 0 & 57 \\
\hline
\end{tabular}




\section{References}

1. Biernacki, J.J.; Bullard, J.W.; Sant, G.; Banthia, N.; Brown, K.; Glasser, F.P.; Jones, S.; Ley, T.; Livingston, R.; Nicoleau, L.; et al. Cements in the 21(st) Century: Challenges, Perspectives, and Opportunities. J. Am. Ceram. Soc. 2017, 100, 2746-2773. [CrossRef]

2. $\mathrm{Hu}, \mathrm{C}$. Nanoindentation as a tool to measure and map mechanical properties of hardened cement pastes. MRS Commun. 2015, 5, 83-87. [CrossRef]

3. Sebastiani, M.; Moscatelli, R.; Ridi, F.; Baglioni, P.; Carassiti, F. High-resolution high-speed nanoindentation mapping of cement pastes: Unravelling the effect of microstructure on the mechanical properties of hydrated phases. Mater. Des. 2016, 97, 372-380. [CrossRef]

4. Bangaru, S.S.; Wang, C.; Hassan, M.; Jeon, H.W.; Ayiluri, T. Estimation of the degree of hydration of concrete through automated machine learning based microstructure analysis - A study on effect of image magnification. Adv. Eng. Inform. 2019, 42, 100975. [CrossRef]

5. Karaxi, E.K.; Kanellopoulou, I.A.; Karatza, A.; Kartsonakis, I.A.; Charitidis, C.A. Fabrication of carbon nanotube-reinforced mortar specimens: Evaluation of mechanical and pressure-sensitive properties. MATEC Web Conf. 2018, 188, 01019. [CrossRef]

6. Gautham, S.; Sasmal, S. Recent Advances in Evaluation of intrinsic mechanical properties of cementitious composites using nanoindentation technique. Constr. Build. Mater. 2019, 223, 883-897. [CrossRef]

7. Hintsala, E.D.; Hangen, U.; Stauffer, D.D. High-Throughput Nanoindentation for Statistical and Spatial Property Determination. Jom 2018, 70, 494-503. [CrossRef]

8. Bock, F.E.; Aydin, R.C.; Cyron, C.J.; Huber, N.; Kalidindi, S.R.; Klusemann, B. A Review of the Application of Machine Learning and Data Mining Approaches in Continuum Materials Mechanics. Front. Mater. 2019, 6. [CrossRef]

9. Koumoulos, E.P.; Tofail, S.A.M.; Silien, C.; De Felicis, D.; Moscatelli, R.; Dragatogiannis, D.A.; Bemporad, E.; Sebastiani, M.; Charitidis, C.A. Metrology and nano-mechanical tests for nano-manufacturing and nano-bio interface: Challenges \& future perspectives. Mater. Des. 2018, 137, 446-462. [CrossRef]

10. Kusne, A.G.; Gao, T.; Mehta, A.; Ke, L.; Nguyen, M.C.; Ho, K.M.; Antropov, V.; Wang, C.Z.; Kramer, M.J.; Long, C.; et al. On-the-fly machine-learning for high-throughput experiments: Search for rare-earth-free permanent magnets. Sci. Rep. 2014, 4, 6367. [CrossRef] [PubMed]

11. Romanos, N.; Kalogerini, M.; Koumoulos, E.P.; Morozinis, A.K.; Sebastiani, M.; Charitidis, C. Innovative Data Management in advanced characterisation: Implications for materials design. Mater. Today Commun. 2019, 20, 100541. [CrossRef]

12. Nieves, J.; Santos, I.; Penya, Y.K.; Rojas, S.; Salazar, M.; Bringas, P.G. Mechanical properties prediction in high-precision foundry production. In Proceedings of the 7th IEEE International Conference on Industrial Informatics (INDIN 2009), Cardiff, UK, 23-26 June 2009; pp. 31-36. [CrossRef]

13. Vignesh, B.; Oliver, W.C.; Kumar, G.S.; Phani, P.S. Critical assessment of high speed nanoindentation mapping technique and data deconvolution on thermal barrier coatings. Mater. Des. 2019, 181, 108084. [CrossRef]

14. Butler, K.T.; Davies, D.W.; Cartwright, H.; Isayev, O.; Walsh, A. Machine learning for molecular and materials science. Nature 2018, 559, 547-555. [CrossRef] [PubMed]

15. Zhu, W.; Hughes, J.J.; Bicanic, N.; Pearce, C.J. Nanoindentation mapping of mechanical properties of cement paste and natural rocks. Mater. Charact. 2007, 58, 1189-1198. [CrossRef]

16. Koumoulos, E.P.; Jagadale, P.; Lorenzi, A.; Tagliaferro, A.; Charitidis, C.A. Evaluation of surface properties of epoxy-nanodiamonds composites. Compos. B Eng. 2015, 80, 27-36. [CrossRef]

17. Sorelli, L.; Constantinides, G.; Ulm, F.-J.; Toutlemonde, F. The nano-mechanical signature of Ultra High Performance Concrete by statistical nanoindentation techniques. Cem. Concr. Res. 2008, 38, 1447-1456. [CrossRef]

18. Li, Y.; Wang, P.; Wang, Z. Evaluation of elastic modulus of cement paste corroded in bring solution with advanced homogenization method. Constr. Build. Mater. 2017, 157, 600-609. [CrossRef]

19. Li, Y.; Zhang, G.; Wang, Z.; Wang, P.; Guan, Z. Integrated experimental-computational approach for evaluating elastic modulus of cement paste corroded in brine solution on microscale. Constr. Build. Mater. 2018, 162, 459-469. [CrossRef]

20. Koumoulos, E.P.; Paraskevoudis, K.; Charitidis, C.A. Constituents Phase Reconstruction through Applied Machine Learning in Nanoindentation Mapping Data of Mortar Surface. J. Compos. Sci. 2019, 3, 63. [CrossRef]

21. Nežerka, V.; Hrbek, V.; Prošek, Z.; Somr, M.; Tesárek, P.; Fládr, J. Micromechanical characterisation and modeling of cement pastes containing waste marble powder. J. Clean. Prod. 2018, 195, 1081-1090. [CrossRef] 
22. Gao, X.; Wei, Y.; Huang, W. Effect of individual phases on multiscale modeling mechanical properties of hardened cement paste. Constr. Build. Mater. 2017, 153, 25-35. [CrossRef]

23. Hu, C.; Li, Z. Micromechanical investigation of Portland cement paste. Constr. Build. Mater. 2014, 71, 44-52. [CrossRef]

24. Hu, C.; Gao, Y.; Zhang, Y.; Li, Z. Statistical nanoindentation technique in application to hardened cement pastes: Influences of material microstructure and analysis method. Constr. Build. Mater. 2016, 113, 306-316. [CrossRef]

25. Alonso, M.C.; García Calvo, J.L.; Hidalgo, A.; Fernández Luco, L. Development and Application of Low- $p H$ Concretes for Structural Purposes in Geological Repository Systems; Woodhead Publishing: Cambridge, UK, 2010; pp. 286-322. [CrossRef]

26. Scrivener, K.L.; Capmas, A. Calcium Aluminate Cements. Elsevier: Oxford, UK, 1998; pp. 713-782. [CrossRef]

27. Ojovan, M.I.; Lee, W.E.; Kalmykov, S.N. Immobilisation of Radioactive Waste in Cement. In An Introduction to Nuclear Waste Immobilisation; Elsevier: Oxford, UK, 2019; pp. 271-303. [CrossRef]

28. Yip, C.K.; van Deventer, J.S.J. Microanalysis of calcium silicate hydrate gel formed within a geopolymeric binder. J. Mater. Sci. 2003, 38, 3851-3860. [CrossRef]

29. Constantinides, G.; Ulm, F.-J. The nanogranular nature of C-S-H. J. Mech. Phys. Solids 2007, 55, 64-90. [CrossRef]

30. Barbhuiya, S.; Chow, P. Nanoscaled Mechanical Properties of Cement Composites Reinforced with Carbon Nanofibers. Materials 2017, 10, 662. [CrossRef]

31. Wilson, W.; Sorelli, L.; Tagnit-Hamou, A. Automated coupling of NanoIndentation and Quantitative Energy-Dispersive Spectroscopy (NI-QEDS): A comprehensive method to disclose the micro-chemo-mechanical properties of cement pastes. Cem. Concr. Res. 2018, 103, 49-65. [CrossRef]

32. Liu, R.; Han, F.; Yan, P. Characteristics of two types of C-S-H gel in hardened complex binder pastes blended with slag. Sci. China Technol. Sci. 2013, 56, 1395-1402. [CrossRef]

33. Gao, Y.; Hu, C.; Zhang, Y.; Li, Z.; Pan, J. Characterisation of the interfacial transition zone in mortars by nanoindentation and scanning electron microscope. Mag. Concr. Res. 2018, 70, 965-972. [CrossRef]

34. Pelisser, F.; Gleize, P.J.P.; Mikowski, A. Effect of the Ca/Si Molar Ratio on the Micro/nanomechanical Properties of Synthetic C-S-H Measured by Nanoindentation. J. Phys. Chem. C 2012, 116, 17219-17227. [CrossRef]

35. Randall, N.X.; Vandamme, M.; Ulm, F.-J. Nanoindentation analysis as a two-dimensional tool for mapping the mechanical properties of complex surfaces. J. Mater. Res. 2011, 24, 679-690. [CrossRef]

36. Howind, T.; Hughes, J.J.; Zhu, W.; Puertas, F.; Goñi Elizalde, S.; Hernandez, M.S.; Guerrero Bustos, A.; Palacios, M.; Dolado, J.S. Mapping of mechanical properties of cement paste microstructures. In Proceedings of the 13th International Congress on the Chemistry of Cement, Madrid, Spain, 3-8 July 2011; p. 309.

37. Constantinides, G.; Ulm, F.-J. The effect of two types of C-S-H on the elasticity of cement-based materials: Results from nanoindentation and micromechanical modeling. Cem. Concr. Res. 2004, 34, 67-80. [CrossRef]

38. Vandamme, M.; Ulm, F.-J.; Fonollosa, P. Nanogranular packing of C-S-H at substochiometric conditions. Cem. Concr. Res. 2010, 40,14-26. [CrossRef]

39. Brown, L.; Allison, P.G.; Sanchez, F. Use of nanoindentation phase characterisation and homogenization to estimate the elastic modulus of heterogeneously decalcified cement pastes. Mater. Des. 2018, 142, 308-318. [CrossRef]

40. Němeček, J. Creep effects in nanoindentation of hydrated phases of cement pastes. Mater. Charact. 2009, 60, 1028-1034. [CrossRef]

41. Nemecek, J. Nanoindentation in Materials Science; Oliver Kurelic: Rijeka, Croatia, 2012. [CrossRef]

42. Göbel, L.; Bos, C.; Schwaiger, R.; Flohr, A.; Osburg, A. Micromechanics-based investigation of the elastic properties of polymer-modified cementitious materials using nanoindentation and semi-analytical modeling. Cem. Concr. Compos. 2018, 88, 100-114. [CrossRef]

43. Lura, P.; Trtik, P.; Münch, B. Validity of recent approaches for statistical nanoindentation of cement pastes. Cem. Concr. Compos. 2011, 33, 457-465. [CrossRef]

44. Ulm, F.-J.; Vandamme, M.; Bobko, C.; Alberto Ortega, J.; Tai, K.; Ortiz, C. Statistical Indentation Techniques for Hydrated Nanocomposites: Concrete, Bone, and Shale. J. Am. Ceram. Soc. 2007, 90, 2677-2692. [CrossRef]

45. Bzdok, D.; Altman, N.; Krzywinski, M. Statistics versus machine learning. Nat. Methods 2018, 15, $233-234$. [CrossRef] 
46. Krakowiak, K.J.; Thomas, J.J.; Musso, S.; James, S.; Akono, A.-T.; Ulm, F.-J. Nano-chemo-mechanical signature of conventional oil-well cement systems: Effects of elevated temperature and curing time. Cem. Concr. Res. 2015, 67, 103-121. [CrossRef]

47. Trompeta, A.-F.; Koklioti, M.A.; Perivoliotis, D.K.; Lynch, I.; Charitidis, C.A. Towards a holistic environmental impact assessment of carbon nanotube growth through chemical vapour deposition. J. Clean. Prod. 2016, 129, 384-394. [CrossRef]

48. Zhang, H.; Šavija, B.; Luković, M.; Schlangen, E. Experimentally informed micromechanical modelling of cement paste: An approach coupling X-ray computed tomography and statistical nanoindentation. Compos. B Eng. 2019, 157, 109-122. [CrossRef]

49. Aggelakopoulou, E.; Bakolas, A.; Moropoulou, A. Design and evaluation of concrete for restoration interventions on Byzantine monuments. J. Cult. Herit. 2018, 34, 166-171. [CrossRef]

50. Dey, A.; Mukhopadhyay, A. Nanoindentation of Brittle Solids; CRC Press: Boca Raton, FL, USA, 2014. [CrossRef]

51. Tzimas, M.; Michopoulos, J.; Po, G.; Reid, A.C.E.; Papanikolaou, S. Inference and Prediction of Nanoindentation Response in FCC Crystals: Methods and Discrete Dislocation Simulation Examples. arXiv, 2019; arXiv:1910.07587.

52. Asch, V.V. Macro- and Micro-Averaged Evaluation Measures; Technical Report; CLiPS: Antwerpen, Belgium, 2013.

53. Koumoulos, E.; Konstantopoulos, G.; Charitidis, C. Applying Machine Learning to Nanoindentation Data of (Nano-) Enhanced Composites. Fibers 2020, 8, 3. [CrossRef]

54. Schober, P.; Boer, C.; Schwarte, L.A. Correlation Coefficients: Appropriate Use and Interpretation. Anesth. Analg. 2018, 126, 1763-1768. [CrossRef] [PubMed]

55. Chang, C.Y.; Hsu, M.T.; Esposito, E.X.; Tseng, Y.J. Oversampling to overcome overfitting: Exploring the relationship between data set composition, molecular descriptors, and predictive modeling methods. J. Chem. Inf. Model. 2013, 53, 958-971. [CrossRef]

56. Bholowalia, P.; Kumar, A. EBK-Means: A Clustering Technique based on Elbow Method and K-Means in WSN. Int. J. Comput. Appl. 2014, 105, 17-24.

57. Lantz, B. Machine Learning with R-Third Edition, 3rd ed.; Naik, V., Ed.; Packt Publishing Ltd.: Birmingham, UK, 2019.

58. Hu, C. Microstructure and mechanical properties of fly ash blended cement pastes. Constr. Build. Mater. 2014, 73, 618-625. [CrossRef]

59. Hu, M.; Chen, Z.; Wang, S.; Guo, D.; Ma, C.; Zhou, Y.; Chen, J.; Laghari, M.; Fazal, S.; Xiao, B.; et al. Thermogravimetric kinetics of lignocellulosic biomass slow pyrolysis using distributed activation energy model, Fraser-Suzuki deconvolution, and iso-conversional method. Energ. Convers. Manag. 2016, 118, 1-11. [CrossRef]

60. Carbon Component Estimation with TGA and Mixture Modelling. Available online: https://smwindecker. github.io/mixchar/articles/Background.html\#background (accessed on 11 October 2019).

61. Hu, C.; Li, Z. A review on the mechanical properties of cement-based materials measured by nanoindentation. Constr. Build. Mater. 2015, 90, 80-90. [CrossRef]

62. Konstantopoulos, G. Probability Distribution analysis from Nanoindentation data. Available online: https: //gkonstanto.shinyapps.io/NIpdf/:2019 (accessed on 11 October 2019).

(C) 2020 by the authors. Licensee MDPI, Basel, Switzerland. This article is an open access article distributed under the terms and conditions of the Creative Commons Attribution (CC BY) license (http://creativecommons.org/licenses/by/4.0/). 